\title{
SARS-CoV-2 infects brain astrocytes of COVID-19 patients and impairs neuronal viability
}

\section{Fernanda Crunfli}

University of Campinas

Victor Corasolla Carregari

University of Campinas

Flavio Protásio Veras

University of São Paulo

Pedro Henrique Vendramini

University of Campinas

Aline Gazzola Fragnani Valença

University of Campinas

André Saraiva Leão Marcelo Antunes

University of Campinas

Caroline Brandão-Teles

University of Campinas

Giuliana da Silva Zuccoli

University of Campinas

Guilherme Reis-de-Oliveira

University of Campinas

Lícia C.Silva-Costa

University of Campinas

Verônica Monteiro Saia-Cereda

University of Campinas

Ana Campos Codo

University of Campinas

Pierina Lorencini Parise

University of Campinas

Daniel A. Toledo-Teixeira

University of Campinas

Gabriela Fabiano de Souza

University of Campinas

Stéfanie Primon Muraro

University of Campinas

Bruno Marcel Silva Melo 
University of Campinas

\section{Glaucia M. Almeida}

University of São Paulo

Egidi Mayara Silva Firmino

University of Campinas

Isadora Marques Paiva University of São Paulo

Bruna Manuella Souza Silva

University of São Paulo

Raíssa Guimarães Ludwig

University of Campinas

Gabriel Palermo Ruiz

University of São Paulo

Thiago Leite Knittel

University of Campinas

Gustavo Gastão Davanzo

University of Campinas

Jaqueline Aline Gerhardt University of Campinas

Patrícia Brito Rodrigues

University of Campinas

Julia Forato

University of Campinas

Mariene Ribeiro Amorim

University of Campinas

Natália Brunetti Silva

University of Campinas

Matheus Cavalheiro Martini

University of Campinas

Maíra Nilson Benatti

University of São Paulo

Sabrina Batah

University of São Paulo

\section{Li Siyuan}

University of São Paulo

Rafael Batista João

University of Campinas

Lucas Scardua Silva

University of Campinas 


\section{Mateus Henrique Nogueira}

University of Campinas

Ítalo Karmann Aventurato

University of Campinas

Mariana Rabelo de Brito

University of Campinas

Marina Alvim

State University of Campinas https://orcid.org/0000-0002-6780-0761

José Roberto da Silva Júnior

University of Campinas

Lívia Liviane Damião

University of Campinas

Maria Ercilia de Paula Castilho Stefano

University of Campinas

lêda Maria Pereira de Sousa

University of Campinas

Elessandra Dias da Rocha

University of Campinas

Solange Maria Gonçalves

University of Campinas

Luiz Henrique Lopes da Silva

University of Campinas

Vanessa Bettini

University of Campinas

Brunno de Campos

School of Medical Sciences, University of Campinas https://orcid.org/0000-0003-1261-8257

Guilherme Ludwing

University of Campinas

Rosa Maria Mendes Viana

University of São Paulo

Ronaldo Martins

University of São Paulo

Andre Schwambach Vieira

University of Campinas - UNICAMP https://orcid.org/0000-0001-5318-8957

José Carlos Alves-Filho

University of Campinas

Eurico Arruda

University of São Paulo

Adriano Sebollela 
University of São Paulo

Fernando Cendes

State University of Campinas https://orcid.org/0000-0001-9336-9568

\section{Fernando Cunha}

University of São Paulo

André Ricardo de Lima Damásio

University of Campinas

Marco Aurélio Ramirez Vinolo

University of Campinas

\section{Carolina Munhoz}

Universidade de Sao Paulo Instituto de Ciencias Biomedicas https://orcid.org/0000-0002-8656-5234

\section{Stevens K. Rehen}

Federal University of Rio de Janeiro-UFRJ

Thais Mauad

University of São Paulo

\section{Amaro Duarte-Neto}

University of São Paulo

Luiz Fernando Ferraz da Silva

University of São Paulo

Marisa Dolhnikoff

University of São Paulo

\section{Paulo Saldiva}

University of São Paulo

Alexandre Fabro

University of São Paulo

Alessandro S. Farias

University of Campinas

Pedro Manoel M. Moraes-Vieira

University of Campinas

José Luiz Proença Módena

University of Campinas

\section{Clarissa Yasuda}

Department of Neurology - University of Campinas

\section{Marcelo A. Mori}

University of Campinas

\section{Thiago Mattar Cunha}

University of São Paulo

Daniel Martins de Souza ( $\sim$ dmsouza@unicamp.br)

University of Campinas 


\section{Article}

Keywords: COVID-19, Anxiety, Cognitive Impairment, Altered Cerebral Cortical Thickness, Histopathology, Neural Stem Cell, Biogenesis

Posted Date: November 13th, 2020

DOl: https://doi.org/10.21203/rs.3.rs-104944/v1

License: (c) (i) This work is licensed under a Creative Commons Attribution 4.0 International License. Read Full License 


\section{SARS-CoV-2 infects brain astrocytes of COVID-19 patients and impairs neuronal viability}

Fernanda Crunfli ${ }^{1 \#}$, Victor Corasolla Carregari ${ }^{1 \#}$, Flavio Protasio Veras ${ }^{2,3 \#}$, Pedro Henrique Vendramini ${ }^{1 \#}$, Aline Gazzola Fragnani Valença ${ }^{1}$, André Saraiva Leão Marcelo Antunes ${ }^{1}$, Caroline Brandão-Teles ${ }^{1}$, Giuliana da Silva Zuccoli $^{1}$, Guilherme Reis-de-Oliveira ${ }^{1}$, Lícia C.Silva-Costa ${ }^{1}$, Verônica Monteiro Saia-Cereda ${ }^{1}$, Ana Campos Codo ${ }^{4}$, Pierina Lorencini Parise ${ }^{5}$, Daniel A. Toledo-Teixeira ${ }^{5}$, Gabriela Fabiano de Souza ${ }^{5}$, Stéfanie Primon Muraro ${ }^{5}$, Bruno Marcel Silva Melo ${ }^{2,3}$, Glaucia M. Almeida ${ }^{6}$, Egidi Mayara Silva Firmino ${ }^{3}$, Isadora Marques Paiva ${ }^{2,3}$, Bruna Manuella Souza Silva ${ }^{2,3}$, Raíssa Guimarães Ludwig ${ }^{7}$, Gabriel Palermo Ruiz ${ }^{7}$, Thiago Leite Knittel ${ }^{7}$, Gustavo Gastão Davanzo ${ }^{4}$, Jaqueline Aline Gerhardt ${ }^{7}$, Patrícia Brito Rodrigues ${ }^{8}$, Julia Forato ${ }^{5}$, Mariene Ribeiro Amorim ${ }^{5}$, Natália Brunetti Silva ${ }^{9}$, Matheus Cavalheiro Martini ${ }^{5}$, Maíra Nilson Benatti ${ }^{10}$, Sabrina Batah ${ }^{10}$, Li Siyuan ${ }^{10}$, Rafael Batista João ${ }^{11}$, Lucas Scardua Silva ${ }^{11}$, Mateus Henrique Nogueira ${ }^{11}$, Ítalo Karmann Aventurato ${ }^{11}$, Mariana Rabelo de Brito ${ }^{11}$ Marina Koutsodontis Machado Alvim ${ }^{11}$, José Roberto da Silva Júnior ${ }^{12}$, Lívia Liviane Damião ${ }^{12}$, Maria Ercilia de Paula Castilho Stefano ${ }^{12}$, Iêda Maria Pereira de Sousa ${ }^{12}$, Elessandra Dias da Rocha ${ }^{12}$, Solange Maria Gonçalves ${ }^{12}$, Luiz Henrique Lopes da Silva ${ }^{12}$, Vanessa Bettini ${ }^{12}$, Brunno Machado de Campos ${ }^{11}$, Guilherme Ludwig $^{13}$, Rosa Maria Mendes Viana ${ }^{14,15}$, Ronaldo Martins ${ }^{14,15}$, Andre S. Vieira ${ }^{16}$, José Carlos Alves-Filho ${ }^{2,3}$, Eurico Arruda ${ }^{14,15}$, Adriano S. Sebollela $^{6}$, Fernando Cendes ${ }^{11}$, Fernando Queiroz Cunha ${ }^{2,3}$, André Damásio ${ }^{7,17}$, Marco Aurélio Ramirez Vinolo ${ }^{8,17,18}$ Carolina Demarchi Munhoz ${ }^{19}$, Stevens K. Rehen ${ }^{20,21}$, Thais Mauad ${ }^{22}$, Amaro Nunes Duarte-Neto ${ }^{22}$, Luiz Fernando Ferraz da Silva ${ }^{22}$, Marisa Dolhnikoff ${ }^{22}$, Paulo Saldiva ${ }^{22}$, Alexandre Todorovic Fabro ${ }^{10}$, Alessandro S. Farias ${ }^{9,17,18,20}$, Pedro Manoel M. Moraes-Vieira ${ }^{4,17,18}$, José Luiz Proença Módena ${ }^{5}$, Clarissa Lin Yasuda ${ }^{11^{*}}$, Marcelo A. Mori ${ }^{7,17,18^{*}}$, Thiago Mattar Cunha ${ }^{2,3 *}$, Daniel Martins-de-Souza ${ }^{1,17,23,24, *}$.

1- Laboratory of Neuroproteomics, Department of Biochemistry and Tissue Biology, Institute of Biology, University of Campinas (UNICAMP), Campinas, São Paulo, Brazil;

2- Center for Research in Inflammatory Diseases (CRID), Ribeirão Preto Medical School (FMRP), University of São Paulo (USP), Ribeirão Preto, São Paulo, Brazil;

3- Department of Pharmacology, Ribeirão Preto Medical School (FMRP), University of São Paulo (USP), Ribeirão Preto, São Paulo, Brazil;

4- Laboratory of Immunometabolism, Department of Genetics, Evolution, Microbiology and Immunology, Institute of Biology, University of Campinas (UNICAMP), São Paulo, Brazil;

5- Laboratory of Emerging Viruses, Department of Genetics, Evolution, Microbiology and Immunology, Institute of Biology, University of Campinas (UNICAMP), São Paulo, Brazil;

6- Department of Biochemistry and Immunology, Ribeirão Preto Medical School (FMRP), University of São Paulo (USP), Ribeirão Preto, São Paulo, Brazil;

7- Department of Biochemistry and Tissue Biology, Institute of Biology, University of Campinas (UNICAMP), Campinas, São Paulo, Brazil;

8- Laboratory of Immunoinflammation, Department of Genetics, Microbiology and Immunology, Institute of Biology, University of Campinas (UNICAMP), Campinas, São Paulo, Brazil;

9- Autoimmune Research Laboratory, Department of Genetics, Microbiology and Immunology, Institute of Biology, University of Campinas (UNICAMP), Campinas, São Paulo, Brazil;

10- Department of Pathology and Legal Medicine, Ribeirão Preto Medical School (FMRP), University of São Paulo (USP), Ribeirão Preto, São Paulo, Brazil; 
11- Laboratory of Neuroimaging, Department of Neurology, University of Campinas (UNICAMP), Campinas, São Paulo, Brazil;

12- Department of Radiology, Clinical Hospital, University of Campinas (UNICAMP), Campinas, São Paulo, Brazil; 13- Institute of Mathematics, Statistics and Scientific Computation,.University of Campinas (UNICAMP), Campinas, São Paulo, Brazil;

14- Virology Research Center, Ribeirão Preto Medical School (FMRP), University of São Paulo (USP), Ribeirão Preto, São Paulo, Brazil;

15- Department of Cell and Molecular Biology, Ribeirão Preto Medical School (FMRP), University of São Paulo (USP), Ribeirão Preto, São Paulo, Brazil.

16- Department of Animal Biology, Institute of Biology, University of Campinas (UNICAMP), Campinas, São Paulo, Brazil;

17- Experimental Medicine Research Cluster (EMRC), University of Campinas (UNICAMP), Campinas, São Paulo, Brazil;

18- Obesity and Comorbidities Research Center (OCRC), University of Campinas (UNICAMP), Campinas, São Paulo, Brazil;

19- Department of Pharmacology, Institute of Biomedical Science, University of São Paulo, São Paulo, Brazil

20- D'Or Institute for Research and Education (IDOR), Rio de Janeiro, Brazil;

21- Institute of Biomedical Science (ICB), Federal University of Rio de Janeiro (UFRJ), Rio de Janeiro, Brazil;

22- Department of Pathology, School of Medicine (FM), University of São Paulo (USP), São Paulo, Brazil;

23- National Institute for Biomarkers in Neuropsychiatry (INBioN), Brazilian National Council for Scientific and Technological Development (CNPq), Brazil;

24- D'Or Institute for Research and Education (IDOR), São Paulo, Brazil;

\# These authors contributed equally to this work.

*Correspondence should be addressed to:

Daniel Martins-de-Souza, PhD: dmsouza@unicamp.br

Thiago Mattar Cunha, PhD: thicunha@fmrp.usp.br

Marcelo A. Mori, PhD: morima@unicamp.br

Clarissa Lin Yasuda, MD, PhD: cyasuda@unicamp.br 


\begin{abstract}
COVID-19 patients may exhibit neuropsychiatric and neurological symptoms. We found that anxiety and cognitive impairment are manifested by $28-56 \%$ of SARS-CoV-2-infected individuals with mild respiratory symptoms and are associated with altered cerebral cortical thickness. Using an independent cohort, we found histopathological signs of brain damage in $25 \%$ of individuals who died of COVID-19. All of the affected brain tissues exhibited foci of SARS-CoV-2 infection and replication, particularly in astrocytes. Infection of neural stem cellderived astrocytes changed energy metabolism, altered key proteins and metabolites used to fuel neurons and for biogenesis of neurotransmitters, and elicited a secretory phenotype that reduces neuronal viability. Our data support the model where SARS-CoV-2 reaches the brain, infects astrocytes and triggers neuropathological changes that contribute to the structural and functional alterations in the brain of COVID-19 patients.
\end{abstract}




\section{Main}

COVID-19 is a disease caused by infection with the severe acute respiratory syndrome coronavirus 2 (SARS-CoV-2). Although the most commonly observed symptoms of COVID-19 are respiratory and related to pulmonary infection, a growing body of evidence has demonstrated that SARS-CoV-2 may have extrapulmonary effects ${ }^{1}$, including the central nervous system (CNS). Notably, over 30\% of hospitalized COVID-19 patients manifest neurological and even neuropsychiatric symptoms ${ }^{2,3}$, eventually presenting some degree of encephalitis ${ }^{4}$. One study revealed that more than half of these hospitalized patients continue to exhibit neurological symptoms even after approximately three months from the acute stage ${ }^{5}$. Impaired cognition was also confirmed in recovered patients after hospitalization ${ }^{6}$. The neurological impairment is consistent with substantial damage to the nervous system ${ }^{7}$.

Previous studies on Severe Acute Respiratory Syndrome (SARS) patients reported the presence of the SARS coronavirus in the brain tissue and cerebrospinal fluid of subjects who presented neurological symptoms ${ }^{8-10}$. SARS-CoV-2 RNA was also detected in the cerebrospinal fluid of patients with meningitis ${ }^{11-13}$. Moreover, alterations in the cerebral cortical region compatible with viral infection ${ }^{14}$, loss of white matter and axonal injury ${ }^{15}$ have all been reported in COVID-19 patients.

In line with the proposed neurotropic properties of the SARS-CoV-2, recent evidence demonstrated the presence of viral proteins in human brain regions of COVID-19 patients and in the brain of K18-ACE2 transgenic mouse infected with SARS-CoV-2 ${ }^{16,17}$. The presence of SARS-CoV-2 in the human brain has been associated with significant astrogliosis, microgliosis and immune cell accumulation ${ }^{16}$. Further indicating the ability of SARS-CoV-2 to infect cells of the CNS, Song and colleagues showed that SARS-CoV-2 was able to infect human brain 
organoid cells in culture. Despite the accumulating evidence, little is known about the cellular and molecular mechanisms involved in SARS-CoV-2 infection of the brain and the consequent repercussion to brain structure and functionality. To gain further insights into the neuropathological and neurological consequences of COVID-19 and possible cellular and molecular mechanisms, we performed a broad translational investigation associating clinical and brain imaging features of COVID-19 patients with neuropathological and biochemical changes caused by SARS-CoV-2 infection on the CNS. We found that astrocytes are the main sites of viral infection and replication at the CNS, causing metabolic alterations consistent with the neuropathological alterations and neuropsychiatric symptoms observed in COVID-19 patients.

\section{Results}

\section{Neuropsychiatric symptoms in convalescent COVID-19 patients correlate with altered cerebral cortical thickness}

To explore the possibility of brain damage in COVID-19 patients and dissociate it from the indirect consequences of the severe stage of the disease, we performed cortical surface-based morphometry analysis using high-resolution 3T MRI of 81 subjects diagnosed with COVID-19 who had mild respiratory symptoms and did not require hospitalization or oxygen support. The analysis was performed within a median interval of 54 days after SARS-CoV-2 detection by RTqPCR and the subjects were compared to 145 healthy volunteers (Supplementary Table 1 and 2). The analysis revealed areas of reduced cortical thickness in the left lingual gyrus, calcarine sulcus - including the cuneus - and olfactory sulcus - including the rectus gyrus (Fig. 1a). In contrast, increased thickness was detected in the central sulcus - including the precentral and postcentral gyrus - and superior occipital gyrus (Fig. 1a), which can be associated with vasogenic 
edemas ${ }^{18}$. A subgroup of these individuals $(n=61)$ were subjected to neuropsychological evaluation for anxiety (Beck Anxiety Inventory, BAI), depression (Beck Depression Inventory, BDI), logical memory (Wechsler Memory Scale), cognitive functions (TRAIL Making Test) and fatigue (Chalder Fatigue Questionnaire, CFQ). These tests were performed between 21 and 120 days after diagnosis (median of 59 days). Symptoms of anxiety were identified in approximately $28 \%$ of the subjects, and $20 \%$ of individuals presented symptoms of depression (Extended Data Fig. 1a). Abnormal performances were observed in nearly $28 \%$ of participants on logical memory and approximately $34 \%$ and $56 \%$ on TRAIL A and B, respectively (Supplementary Table 3 and Extended Data Fig. 1b). We also correlated the changes in cortex thickness with the neuropsychological evaluation. We identified a negative correlation between BAI and cortical thickness of orbitofrontal regions (adjusted for CFQ) (Fig. 1b and Supplementary Table 4) and a positive correlation between TRAIL B and cortical thickness of the right gyrus rectus (Fig. 1c and Supplementary Table 5). We additionally identified significant partial correlations between logical memory (immediate recall test, adjusted for BAI, BDI and CFQ) and cortical thickness of regions associated with language (Supplementary Table 6). These results suggest that a thinner cortex in these areas is associated with poor performance on this verbal memory task. Overall, our findings indicated major alterations in cortical structure associated with neuropsychiatric symptoms as a consequence of COVID-19 even in patients with mild or no respiratory symptoms.

\section{SARS-CoV-2 infects and replicates in human brain astrocytes of COVID-19 patients}

Brain alterations in COVID-19 patients could be a consequence of inflammatory or hemodynamic changes secondary to peripheral infection or could be caused by the ability of SARS-CoV-2 to invade the CNS and compromise cell viability and brain function. Although 
exacerbated inflammation and cardiovascular dysfunction have been well-characterized in COVID-19 patients who progress to the severe stages of the disease ${ }^{19}$, the degree of infection of the CNS by SARS-CoV-2 remains elusive. In order to gain knowledge about this possibility, we performed a minimally invasive autopsy via endonasal trans-ethmoidal access to obtain brain samples from 26 individuals who died of COVID-19. Initially, we performed an unbiased histopathological analysis of hematoxylin and eosin-stained brain sections in order to find features of brain alterations. These analyses revealed alterations consistent with necrosis and inflammation in $19.2 \%$ of the brain tissues from these individuals (5 out of 26) (Fig. 2a and Extended Data Fig. 2a). Notably, SARS-CoV-2 genetic material and spike protein were detected in all of these five samples (Fig. 2b,c). SARS-CoV-2 spike protein was detected in about one third of the cells in one of the slices of brain tissue analyzed (Fig. 2d), the majority of these cells being astrocytes (GFAP+ cells; Fig. 2e). We also detected SARS-CoV-2 spike protein in neurons $(\mathrm{NeuN}+$ cells; Fig. 2e and Extended Data Fig. 2b), but not in microglia (Iba-1+ cells; Fig. 2e and Extended Data Fig. 2c). The specificity of anti-spike antibodies was validated in brain tissue of COVID-19 free patients and in SARS-CoV-2-infected Vero cells (Extended Data Fig. 3a-b). Additionally, the presence of SARS-CoV-2 spike protein correlated with the presence of doublestranded RNA (dsRNA) in the cells (Fig. 2c, f), indicating replicative virus in the brain tissue ${ }^{20}$.

In order to confirm the susceptibility of human astrocytes to SARS-CoV-2 infection, neural stem cell-derived human astrocytes were exposed to the virus for $1 \mathrm{~h}$, washed thoroughly and analyzed after 24h (Fig 3a). We confirmed that SARS-CoV-2 may infect human astrocytes (Fig. 3b-e) as the viral genetic material (Fig. 3b) and the spike protein were detected in infected cells (Fig. 3c,d). Notably, we also found the presence of dsRNA in SARS-CoV-2-infected astrocytes in vitro, but not in mock-infected control cells (Fig. 3c,e). Altogether, these results 
indicate that human astrocytes are permissive cells for SARS-CoV-2 infection and represent a site for virus replication in the CNS.

\section{Proteomic and metabolomic changes in SARS-CoV-2-infected human astrocytes}

In an attempt to identify downstream mechanisms triggered by SARS-CoV-2 infection of astrocytes and possibly involved in brain changes observed in COVID-19 patients, we employed an unbiased proteomic analysis of infected human astrocytes (Fig. 3a). Liquid chromatographymass spectrometry (LC/MS)-based shotgun proteomics revealed 233 differentially expressed proteins in SARS-CoV-2-infected astrocytes compared to mock-control cells, being 88 upregulated and 145 downregulated (Extended data fig. 4a). A heatmap constructed based on the relative abundance of each protein showed a group of approximately 50 proteins of which the pattern of expression formed a molecular signature that distinguished infected astrocytes from mock-controls (Fig. 4a). Pathway enrichment analysis and interactome analysis revealed that these proteins are involved in a wide range of biological processes and predicted regulatory networks affected by SARS-CoV-2 infection (Fig. 4b). Pathways involved in carbon metabolism are among the most enriched and include "Glycolysis/Gluconeogenesis" and the "Pentose Phosphate Pathway", indicating altered glucose metabolism (Fig. 4c).

We have also conducted LC/MS proteomics with a different set of samples consisting of 12 postmortem brain samples from COVID-19 patients vs. 8 SARS-CoV-2-negative controls. We identified 119 differentially expressed proteins with the most highly enriched pathways being associated with neurodegenerative diseases and carbon metabolism (Extended data fig. 4b). Notably, astrocyte proteins were enriched among the differentially expressed proteins, consistent with the higher frequency of infected astrocytes (Fig. 4d). Moreover, proteins found 
differentially expressed in SARS-CoV-2-infected astrocytes and in postmortem brain tissue samples were commonly associated with glycolysis/gluconeogenesis, biosynthesis of amino acids, the pentose phosphate pathway and necroptosis (Fig. 4e). These analyzes indicate that SARS-CoV-2 infects astrocytes in CNS and leads to neurodegeneration and changes in carbon metabolism.

Because metabolic pathways appeared altered in our proteomic analysis, we sought to investigate whether the infection of human astrocytes would impact the levels of key metabolites involved in energy metabolism. LC/MS-based metabolomic analysis of SARS-CoV-2-infected astrocytes showed marked changes in metabolic intermediates of glycolysis and anaplerotic reactions, indicating extensive remodeling of astrocyte metabolism (Fig. 5a). This phenomenon was marked by the decrease in pyruvate and lactate, which are downstream metabolites of the glycolytic pathway, as well as a reduction in glutamine and intermediates of glutamine metabolism such as glutamate, GABA and alpha-ketoglutarate (Fig. 5a). On the other hand, there were no significant changes in the tricarboxylic acid cycle (TCA cycle) intermediates (Extended data Fig. 5). Astrocyte bioenergetics was further characterized by Seahorse Extracellular Flux analysis, showing increased respiration in infected cells (Fig. 5b). This was because of an increase in both mitochondrial and non-mitochondrial oxygen consumption; the former was linked to higher proton leak as well as increased ATP synthesis and mitochondrial spare capacity. Together, these results demonstrate increased metabolic activity in SARS-CoV-2infected astrocytes and a reduction of metabolites used by these cells to support neuronal metabolism and function. 


\section{Conditioned medium of SARS-CoV-2-infected astrocytes reduces neuronal viability}

Astrocytes are essential in the control of brain homeostasis not only because they are the main energy reservoirs of the brain ${ }^{21}$ but also due to their important role in the protective response to cell damage triggered by infection or sterile inflammation ${ }^{22,23}$. There is evidence that astrocytes secrete undetermined neurotoxic factors ${ }^{22-24}$ and are also involved in the uptake and distribution of brain metabolites ${ }^{25,26}$. Thus, we investigated whether neuronal viability could be indirectly affected by a conditional medium from SARS-CoV-2-infected astrocytes. To test that, we cultured differentiated SH-SY5Y neurons with the conditioned medium of SARS-CoV-2infected astrocytes or control medium (Fig. 6a). SH-SY5Y cells are more closely related to adrenergic neurons, but they also express dopaminergic markers ${ }^{27}$. Conditioned medium from SARS-CoV-2-infected astrocytes reduced in 16\% the viability of SH-SY5Y neurons while increasing by about 3-fold the rate of apoptosis (Fig. 6b). Notably, the direct infection of SHSY5Y neurons did not reduce cell viability (Fig. 6b,c). In addition, SARS-CoV-2 genetic material was not significantly detected in SH-SY5Y neurons incubated with medium from SARS-CoV-2-infected astrocytes (Fig. 6c), ruling out that the cause of neuronal death could be due to infectious particles present in the conditioned medium. These results suggest that the infection of astrocytes by SARS-CoV-2 leads to the release of a soluble factor that reduces neuronal viability.

\section{Discussion}

Our study evidence structural and functional alterations in the brain tissue of COVID-19 patients as a consequence of astrocyte infection by SARS-CoV-2. This study and other reports showing alterations in brain structure and the manifestation of neurological symptoms in 
COVID-19 patients ${ }^{28,29}$ raise a debate on whether these clinical features are consequence of peripheral changes or rather the potential ability of the virus to invade the CNS. Our findings support the latter, at least in part, as we detected SARS-CoV-2 in the brain tissue collected from patients who died of COVID-19 and neuropsychiatric manifestations in patients with mild or no respiratory symptoms. The potential of SARS-CoV-2 to infect brain cells has been demonstrated using in vitro models such as stem cell-derived neural cells and cerebral organoids ${ }^{17}$. Viral particles have also been found in the brain ${ }^{16}$, localized in the microvasculature and in neurons ${ }^{17}$, as well as in the choroid plexus ${ }^{30}$ and meninges ${ }^{31}$. However, the magnitude of this infection and its distribution in the brain tissue has not been demonstrated yet. We show that SARS-CoV-2 may infect almost one third of the cells in the brain and the majority of these cells are astrocytes.

Cell marker analysis of proteins differentially expressed in postmortem brain tissue from COVID-19 patients revealed an enrichment of astrocyte proteins, suggesting that these cells are indeed the most affected by SARS-CoV-2 infection. Our proteomics data also highlighted changes in components of carbon metabolism pathways in both in vitro infected astrocytes and postmortem brain tissues from COVID-19 patients. These pathways were particularly associated with glucose metabolism.

Since astrocyte metabolism is key to support neuronal function, we hypothesized that these changes could indirectly impact neurons. Astrocytes support neurons metabolically by exporting lactate ${ }^{32}$. One of the most critical alterations caused by SARS-CoV-2 infection in astrocytes is the decrease in pyruvate and lactate levels. Moreover, intermediates of glutamine metabolism such as glutamate and GABA are decreased in SARS-CoV-2-infected astrocytes. On the other hand, there were no significant changes in the intermediates of the TCA cycle. Together with the increased oxygen consumption rate in SARS-CoV-2-infected astrocytes, these 
results suggest that glycolysis and glutaminolysis are being used to fuel carbons into the TCA cycle to sustain the increased oxidative metabolism of infected astrocytes. Importantly, astrocyte-derived lactate and glutamine are required for neuronal metabolism ${ }^{26,33}$ and synthesis of neurotransmitters such as glutamate and GABA, respectively ${ }^{34}$. Astrocytes play a vital role in neurotransmitter recycling, a crucial process for the maintenance of synaptic transmission and neuronal excitability. This is especially important for glutamatergic synapses since proper glutamate uptake by astroglia prevents the occurrence of excitotoxicity ${ }^{35}$. Upon this uptake, glutamine synthetase converts glutamate to glutamine, which can then be transferred back to neurons, thus closing the glutamate-glutamine cycle. This is also true for GABAergic synapses, where the neurotransmitter GABA is taken up by astrocytes and metabolized first to glutamate and then to glutamine ${ }^{36}$. Moreover, astrocytes are responsible for maintaining glutamate levels in the brain. Hence, given the importance of the coupling between astrocytes and neurons, astrocytic alterations in glucose and glutamine metabolism are expected to compromise neuronal function, affecting neuronal metabolism and synaptic function and plasticity ${ }^{37}$.

We also found that SARS-CoV-2 infection elicits a secretory phenotype in astrocytes that results in increased neuronal apoptosis. Neuronal death may explain, at least partially, the alterations in cortical thickness found in COVID-19 patients. A recent study with 60 recovered patients and 39 healthy controls also identified gray matter abnormalities 97 days after the onset of the disease, with increased volume in some areas of the brain ${ }^{5}$. While that study analyzed hospitalized patients, we evaluated individuals that did not have to be hospitalized (i.e., had mild respiratory symptoms), and nevertheless, we observed notable alterations of cortical thickness. Importantly, some of these alterations correlated with symptoms of anxiety and impaired cognition, which is consistent with previous literature ${ }^{38,39}$. Since one of the hypotheses for the 
neuroinvasive mechanism of SARS-CoV-2 is via the olfactory nerves ${ }^{40}$, we speculate that the associations between BAI and TRAIL B scores and structural alterations in the orbitofrontal region may be a result of the action of the virus in this cortical area, closely related to the olfactory nerves.

Our findings are consistent with a model in which SARS-CoV-2 is able to reach the CNS of COVID-19 patients, infects astrocytes and secondarily impairs neuronal function and viability. These changes are likely to contribute to the alterations of brain structure as observed here and elsewhere, thereby resulting in the neurological and neuropsychiatric symptoms manifested by some COVID-19 patients. Our study comes as a cautionary note that interventions directed to treat COVID-19 should also envision ways to prevent SARS-CoV-2 invasion of the $\mathrm{CNS}$ and/or replication in astrocytes.

\section{Material and Methods}

\section{Brain imaging and neuropsychological evaluation}

\section{Participants}

Eighty-one patients (60 women, median 37 years of age) previously infected with SARSCoV-2 were enrolled prospectively for this study after signing an informed consent form approved by the local Ethics Committee. These individuals presented mild symptoms during the acute phase and did not require hospitalization or oxygen therapy. They had a median interval of 54 days (range 16-120 days) between their RT-PCR exam and the day of MRI scanning and interview. For cortical thickness analysis, we included one hundred and forty-five controls (103 women, median 38 years of age) ${ }^{41}$ from our Neuroimaging databank, given the difficulties and 
risk of recruiting healthy volunteers during the pandemic. The outpatients and healthy controls were balanced for age $(\mathrm{p}=0.45)$ and $\operatorname{sex}(\mathrm{p}=0.65)$. The neuropsychological evaluations and neuroimaging analyses were approved by the Research Ethics Committee of the University of Campinas (CAAE: 31556920.0 .0000 .5404$)$ and all subjects signed a consent form to participate.

\section{Neuropsychological evaluation}

We performed neuropsychological evaluations of sixty-one of these patients. They were tested for symptoms of anxiety using the Beck Anxiety Inventory (BAI) and symptoms of depression using the Beck Depression Inventory (BDI) ${ }^{42}$. Symptoms of anxiety were confirmed for those with a BAI higher than 10 points, and depression symptoms defined for those with minimum of 14 points on the BDI. In terms of depression, subjects were categorized with mild (BDI 14-19), moderate (20-35) and severe (36-63) symptoms. Anxiety symptoms were considered mild (BAI 11-19), moderate (20-30) and severe (31-63). We evaluated verbal memory (immediate and delayed episodic memory) using the Logical Memory subtest from the Wechsler Memory Scale (WMS-R) ${ }^{43}$, in which the examiner verbally presents two stories, and each story includes 25 pertinent pieces of information. Subjects are required to recall details of each story immediately after its presentation and again after 20 minutes. To evaluate other cognitive functions, we applied the TRAIL Making Test (TMT), which is subdivided into two steps. Step $A$ assesses processing speed and visual search in a task that requires ascending connection order of 25 numbers, randomly arranged. Step B evaluates alternating attention and cognitive flexibility in a task associated with shifting rules in an ascending sequence of 25 numbers. A training stage is applied to both steps. We calculated z-scores for the results of these tests based on Brazilian normative data ${ }^{43,44}$. For each test, the function was categorized as: "preserved" if the z-score was higher than -0.99; "below average" when the z-score was between 
-1 and -1.49 ; "minor impairment" if the z-score was between -1.5 and -1.99 ; and "major impairment" for z-score values equal to or lower than -2. The Chalder Fatigue Questionnaire (CFQ-11) ${ }^{45}$ was used to evaluate fatigue in these subjects; they were instructed to answer 11 questions (measured on a Likert scale 0-3), which yields a global score out of 33 .

\section{Neuroimaging analysis}

We obtained the structural, 3D, T1-weighted images from a 3T Achieva-Philips MRI scanner (voxel size: $1 \times 1 \times 1 \mathrm{~mm}, \mathrm{TE}=3.2 \mathrm{~ms}, \mathrm{TR}=7 \mathrm{~ms}$, matrix $=240 \times 240 \times 180$, flip angle $=8$ and $\left.\mathrm{FOV}=240 \times 240 \mathrm{~mm}^{2}\right)^{41,42}$. We performed imaging analysis with the CAT12 toolbox (http://www.neuro.uni-jena.de/cat/, version r1711) within SPM12

(http://www.fil.ion.ucl.ac.uk/spm/, version 7487) using MATLAB 2017b to extract Cortical Thickness (CT) maps, according to the default parameters. The T1 images were initially spatially registered and segmented into gray matter, white matter and cerebrospinal fluid. Finally, we calculated the cortical thickness using the projection method described by Dahnke et al. ${ }^{46}$. For voxelwise analysis of extracted maps, we used CAT12/SPM12 tools for an independent T-test (comparing COVID-19 patients and healthy controls), including age and sex as covariates. The

results displayed were corrected for multiple comparisons using False Discovery Rate (FDR) ${ }^{47}$ correction $(p<0.05)$. For anatomical identification, we used the "Destrieux Atlas 2009" 48. Cortical parcellation was performed with standard CAT12 tools to extract the cortical thickness of regions of interest for correlations with neuropsychological scores. We used SPSS 22 for statistical analysis of clinical and neuropsychological variables. The FDR procedure was applied to adjust p-values for multiple comparisons (when necessary) with R software ${ }^{49}$. 


\section{Postmortem brain samples from COVID-19}

Twenty-six individuals who died from complications related to COVID-19 were autopsied with an ultrasound-guided, minimally invasive approach using endonasal transethmoidal access. Brain tissue samples were collected and fixed using a 10\% neutral buffered formalin solution. After fixation, the tissue was embedded in a paraffin block and sectioned into slices with a thickness of $3 \mu \mathrm{m}$. The sections were stained by H\&E and immunofluorescence. For proteomic analysis, twelve COVID-19 patients were autopsied using the same approach. Brain tissue samples were collected and macerated in a lysis buffer $(100 \mathrm{mM}$ Tris- $\mathrm{HCl}, \mathrm{pH} 8.0,150$ $\mathrm{mM} \mathrm{NaCl}, 1 \mathrm{mM}$ EDTA, $0.5 \%$ Triton X-100) prior to trypsin digestion. The autopsy studies were approved by the National Commission for Research Ethics (CAAE: 32475220.5.0000.5440 and CAAE: 38071420.0.1001.5404).

\section{Generation of human astrocytes (hES-derived)}

Differentiation of glial progenitor cells was performed from neural stem cells (NSC) derived from pluripotent human embryonic stem cells (hES, cell line BR-1) ${ }^{50}$, according to the method published by Trindade, $2020{ }^{51}$. NSCs were cultured in plates coated with Geltrex Matrix (Thermo Fisher Scientific, MA, USA) using 1:1 NeurobasalTM/Advanced DMEM/F12 medium and $2 \%$ neural induction supplement. Upon reaching $50 \%$ confluence, the medium was changed to DMEM/F12, 1\% N2 supplement, 1\% fetal bovine serum (FBS), and 1\% PenicillinStreptomycin and maintained at $37^{\circ} \mathrm{C}$ in humidified air with $5 \% \mathrm{CO}_{2}$ for 21 days. At this stage, cells were considered glial progenitor cells (GPCs). Subsequently, GPCs were plated at low density (30-40\% confluence) on Geltrex coated plates and treated with DMEM/F12 medium, 1\% GlutaMAX Supplement, 10\% FBS and 1\% penicillin-streptomycin. The differentiation medium 
was replaced every 2-3 days. After 4 weeks of differentiation, the cells were considered mature astrocytes. The cells were plated on Geltrex-coated coverslips at a density of $4 \times 10^{4}$ cells for immunostaining assays; and $25 \times 10^{4}$ cells for viral load, proteomic and metabolomic analysis. All products used for cell culture are from Thermo Fisher Scientific, MA, USA.

\section{Virus strain}

The HIAE-02-SARS-CoV-2/SP02/human/2020/BRA (GenBank accession number MT126808.1) virus strain was used for all in vitro experiments. The virus was isolated from the first confirmed case of COVID-19 in Brazil and kindly donated by Prof. Dr. Edison Durigon (ICB-USP). Viral stock was propagated in Vero CCL-81 cells (ATCC) cultivated in DMEM supplemented with $10 \%$ heat-inactivated FBS and $1 \%$ of penicillin and streptomycin, and incubated at $37^{\circ} \mathrm{C}$ with $5 \% \mathrm{CO}_{2}$ atmosphere. Viral titer was determined by the plaque-forming assay using Vero cells.

\section{In vitro infection}

Astrocytes were infected with SARS-CoV-2 for 1 hour using an MOI of 0.1 (proteomics, metabolomics, gene expression, viral load, bioenergetics assays and flow cytometer analysis) and 1 (immunostaining experiments) under gentle agitation at room temperature. After viral adsorption, cells were washed twice with phosphate-buffered saline (PBS) and incubated with DMEM/F12 supplemented with 10\% FBS, $1 \%$ GlutaMAX and 1\% penicillin and streptomycin for 24 hours at standard culture conditions $\left(37^{\circ} \mathrm{C}\right.$ and $5 \% \mathrm{CO}_{2}$ atmosphere). Astrocytes cells were harvested according to the experiment: immunostaining, proteomics and metabolomic analysis, gene expression analysis and viral load, bioenergetics test and flow cytometer analysis. 


\section{Immunostaining and confocal microscopy}

Brain sections from autopsies and astrocyte cell cultures were fixed with $10 \%$ neutral buffered formalin solution or 4\% paraformaldehyde (PFA), respectively. Subsequently, the samples were incubated with primary antibodies: mouse monoclonal anti-GFAP Alexa Fluor 488 (EMD Millipore, clone GA5, cat. MAB3402X, 1:400), human chimeric monoclonal anti-SARSCoV-2 Spike S1 (GeneScript, clone HC2001, cat. A02038, 1:500), rabbit polyclonal anti-SARSCoV-2 Spike (Rhea Biotech, cat. IM-0828, 1:200), mouse monoclonal anti-double stranded RNA J2 (dsRNA, SCICONS English \& Scientific Consulting Kft., clone J2-1909, cat. 10010200; 1:1,000), rabbit polyclonal anti-IBA1 (FUJIFILM Wako Pure Chemical Corporation, cat. 01919741, 1:1,000) and rabbit monoclonal anti-NeuN (Abcam, clone EPR12763, cat. Ab128886, 1:1,000). The slides were washed twice with TBS-T (Tris-Buffered Saline with Tween 20) and incubated with secondary antibodies donkey anti-mouse IgG AlexaFluor 647 (Thermo Fisher Scientific, cat. A32787; 1:800) or AlexaFluor 488 (Abcam; cat. ab150061; 1:800), donkey antirabbit IgG AlexaFluor 488 (Abcam; cat. ab150065; 1:800) or AlexaFluor 594 (Abcam; cat. ab150076; 1:800) and goat anti-human IgG FITC (Rhea Biotech, cat. IC-3H04, 1:400). Controls were performed for secondary antibody fluorescence. Antibodies for detecting SARS-CoV-2 in human brain tissue and human astrocytes in vitro were first validated on SARS-CoV-2-infected and non-infected Vero cells. In addition, we validated the antibodies in brain sections from control (Non-COVID-19).

Nuclei were stained with DAPI (Life Technologies, cat. D1306; 1:1,000). Images were acquired by an Axio Observer combined with an LSM 780 confocal device (Carl Zeiss) with 630x magnification and Z-stack $(0.15 \mu \mathrm{m})$ for brain sections. Colocalization analysis between GFAP and SARS-CoV-2 S1 or GFAP and dsRNA were quantified using the Fiji/ImageJ 
software. To determine colocalization, we used a ratio of GFAP:SARS-CoV-2 and

GFAP:dsRNA in each sample by analyzing Pearson's correlation coefficient.

\section{Proteomics sample preparation, LC-MS/MS analysis and data processing}

Astrocytes infected with SARS-CoV-2 and a mock control were collected in biological

triplicates. Cells were chemically lysed with Lysis Buffer: (100 mM Tris-HCl, 1 mM EDTA, 150 $\mathrm{mM} \mathrm{NaCl}, 1 \%$ Triton-X, protease and phosphatase inhibitors) and mechanically lysed with an ultrasonication probe in 3 cycles of 20 s each with $90 \%$ of frequency. The total protein extract was quantified by BCA, according to the manufacturer's instructions (Thermo Fisher Scientific, MA, USA). $30 \mu \mathrm{g}$ of total protein extract from each sample was transferred to a Microcon-10 Centrifugal Filter, with $10 \mathrm{kDa}$ cutoff, for FASP protein digestion ${ }^{52}$. Proteins were reduced (10 $\mathrm{mM}$ DTT), alkylated (50 mM IAA) and digested overnight by trypsin at $37^{\circ} \mathrm{C}$ in $50 \mathrm{mM}$ ammonium bicarbonate $(\mathrm{AmBic}), \mathrm{pH}$ 8.0. One the following day the peptides were recovered from the filter in $50 \mathrm{mM} \mathrm{AmBic}$, and trypsin activity was quenched by adding formic acid (FA) to a final concentration of $1 \%(\mathrm{v} / \mathrm{v})$, after that the peptides were concentrated in a SpeedVac and stored at $-80^{\circ} \mathrm{C}$ until use.

Digested peptides were resuspended in $0.1 \%$ FA. LC-MS/MS analysis was performed in an ACQUITY M-Class System (Waters Corporation, Milford, MA) coupled online to a Synapt G2-Si mass spectrometer (Waters Corporation, Milford, MA). $1 \mu \mathrm{g}$ of peptides were loaded onto a trapping column (Symmetry C18 $5 \mu \mathrm{m}, 180 \mu \mathrm{m} \times 20 \mathrm{~mm}$, Waters Corporation, Milford, MA) and subsequently separated in the analytical column (HSS T3 C18 $1.8 \mu \mathrm{m}, 75 \mu \mathrm{m} \times 150 \mathrm{~mm}$; Waters Corporation, Milford, MA). For gradient elution, $0.1 \%$ FA was used as eluent A and Acetonitrile-FA (99.9\% ACN:0.1\% FA) as eluent B. Reversed phase gradient was carried out 
over 120 minutes, with a linear gradient $3-60 \%$ acetonitrile over $90 \mathrm{~min}$ at $300 \mathrm{~nL} / \mathrm{min}$. In the Synapt G2-Si, the peptide spectra were acquired by ion mobility-enhanced data-independent acquisition $\left(\mathrm{HDMS}^{\mathrm{e}}\right)$. Mass spectrometry analysis was performed in "Resolution Mode", switching between low $(4 \mathrm{eV})$ and high $(25-60 \mathrm{eV})$ collision energies, using a scan time of $1.0 \mathrm{~s}$ per function over $50-2000 \mathrm{~m} / \mathrm{z}$. The wave velocity for ion mobility separation was $1.000 \mathrm{~m} / \mathrm{s}$ and the transfer wave velocity was $175 \mathrm{~m} / \mathrm{s}$. A [Glu1]-Fibrinopeptide B Standard (Waters Corporation, Milford, MA) was used as the reference lock mass compound. Each sample was run in three technical replicates.

The raw data from each experiment were processed in Progenesis QI for proteomics (Waters Corporation, Milford, MA). Tandem mass spectra were searched against the Homo sapiens proteome database (UNIPROT Protein reviewed release 2020-04), using tolerance parameters of $20 \mathrm{ppm}$ for precursor ions and $10 \mathrm{ppm}$ for product ions. For peptide identification, carbamidomethylation of cysteines was set as a fixed modification, oxidation of methionines as a variable modification, 2 missed cleavages, and a false discovery rate $(\mathrm{FDR})<1 \%$. Protein identification was performed using a minimum of 1 fragment ion matched per peptide, a minimum of 3 fragment ions per protein and a minimum of 1 peptide per protein.

Label-free quantitative analysis was carried out using the relative abundance intensity normalized by all peptides identified. The expression analysis was performed considering the technical replicates for each experimental condition, following the hypothesis that each group is independent. Proteins with ANOVA $(\mathrm{p}) \leq 0.05$ between the groups were considered differentially expressed. 


\section{Bioinformatic Analysis}

Proteomic data visualization was performed in house with Python programming language (v. 3.7.3). Proteins differentially regulated $(\mathrm{p}<0.05)$ were submitted to systems biology analysis in R (v. 4.0) and Cytoscape environments ${ }^{53}$. While performing Over Representation Analysis (ORA), proteins were enriched using ClusterProfiler R package ${ }^{54}$, CellMarker Database ${ }^{55}$, and Kyoto Encyclopedia of Genes and Genomes $\left(\mathrm{KEGG}^{56}\right)$. For the Network analysis, it was run by Reactome Cytoscape Plugin ${ }^{57}$ for module detection and enrichment pathway analysis.

\section{Metabolomics sample preparation, UPLC-MS/MS analysis and data processing}

The medium was washed twice with PBS at physiologic $\mathrm{pH}$, then the cells were collected with $600 \mu \mathrm{L}$ of methanol. Samples were dried and stored at $-80{ }^{\circ} \mathrm{C}$ until the metabolite extraction step. Volumes of $473 \mu \mathrm{L}$ of water, $600 \mu \mathrm{L}$ of methanol, and $1168 \mu \mathrm{L}$ of chloroform were added, and then the tubes were shaken vigorously for 2 minutes. Subsequently, samples were centrifuged for 5 minutes at 13,000 x g. The aqueous supernatant and the organic phase (lower phase) were collected and dried for 60 and 40 min (respectively) in a vacuum concentrator. All samples were stored at $-80{ }^{\circ} \mathrm{C}$ until analysis by UPLC-MS/MS.

The samples were resuspended in $100 \mu \mathrm{L}$ of methanol: water mixture (1:1) and for each analysis, $4 \mu \mathrm{L}$ of the sample was injected. Sample separation was performed by hydrophobic interaction liquid chromatography (HILIC) using an Acquity UPLC® BEH amide column (1.7 $\mu \mathrm{m}, 2.1 \mathrm{~mm} \times 100 \mathrm{~mm})$. The mobile phases used for the separations were $\mathrm{ACN}: \mathrm{H}_{2} \mathrm{O}(80: 20)$ as mobile phase A and $\mathrm{ACN}: \mathrm{H}_{2} \mathrm{O}(30: 70)$ as mobile phase $\mathrm{B}$, both phases containing $10 \mathrm{mM}$ of ammonium formate and $0.1 \%$ of ammonium hydroxide. The separation was then performed by a gradient from $99 \%$ to $1 \%$ buffer A over 7 min. The column was returned to $99 \%$ buffer A for 2 
min for re-equilibration before the next injection for a total run time of $10 \mathrm{~min}$. Data acquisition was performed in negative mode and the instrument was operated in $\mathrm{MS}^{\mathrm{e}}$ mode in the $\mathrm{m} / \mathrm{z}$ range of 50-800 Da, with an acquisition time of $0.1 \mathrm{~s}$ per scan.

The raw files were preprocessed by Progenesis QI software by Waters ${ }^{\circledR}$, and identification was executed using multiple data banks. Identification of the metabolites of interest was carried out manually by spectral features, and the identification level 3 was obtained according to Schrimpe-Rutledge et al. 58 using 5ppm as the error cutoff. The integration area of each peak was used to calculate the violin plot graph and an unpaired t-test with Welch's correction was used for statistical comparison. All analyses were performed using GraphPad Prism 8.0 software (San Diego, CA, USA) and a significance level of $\mathrm{p} \leq 0.05$ was adopted.

\section{RNA extraction, gene expression analysis and viral load}

Total RNA extraction was performed using TRI Reagent according to the manufacturer's instructions (Sigma, St Louis, USA). RNA concentration was determined by a DeNovix spectrophotometer and RNA integrity was assessed by visualization of $28 \mathrm{~S}$ and $18 \mathrm{~S}$ ribosomal RNA on a $1 \%$ agarose gel. Reverse transcription was performed with $0.5 \mu \mathrm{g}$ of RNA using a GoScript Reverse Transcriptase Kit (Promega, Madison, WI, USA) according to the manufacturer's instructions. qPCR was performed using astrocyte cDNA diluted 1:10 and the qPCR SybrGreen Supermix (Qiagen, Valencia, CA, USA) containing forward and reverse primers in RNAse-free water. All reactions were performed in a CFX384 Touch Real-Time PCR Detection System (Biorad, Hercules, CA, USA) and cycling conditions were set as follows: $50^{\circ} \mathrm{C}$ for $2 \mathrm{~min} ; 95^{\circ} \mathrm{C}$ for $10 \mathrm{~min}$; $\left(95^{\circ} \mathrm{C}\right.$ for $15 \mathrm{~s} ; 60^{\circ} \mathrm{C}$ for $\left.1 \mathrm{~min}\right)$ x 40 cycles. To evaluate primer specificity, a melting curve analysis was performed by heating samples from $65^{\circ} \mathrm{C}$ to $99^{\circ} \mathrm{C}\left(1^{\circ} \mathrm{C}\right.$ 
increment changes at $5 \mathrm{~s}$ intervals). All sample measurements were performed in duplicate. Primers were designed with PrimerBlast and used at the concentration of $200 \mathrm{nM}$. Data were normalized to the expression of 18S (Fwd 5' CCCAACTTCTTAGAGGGACAAG 3'; Rev 5' CATCTAAGGGCATCACAGACC 3') and the relative quantification value of each target gene was determined using a comparative CT method ${ }^{59}$. For virus detection, SARS-CoV-2 nucleocapsid N1 primers were used as previously described (Fwd 5'

CAATGCTGCAATCGTGCTAC 3'; Rev 5' GTTGCGACTACGTGATGAGG 3') ${ }^{60,61}$. A serial dilution of SARS-CoV-2 was used as a standard curve. Data were expressed as mean $\pm \mathrm{SEM}$. Statistical significance analysis was calculated by two-tailed unpaired Student's t-test. All analyses were performed using GraphPad Prism 8.0 (San Diego, CA, USA) and a significance level of $\mathrm{p} \leq 0.05$ was adopted.

\section{Astrocyte bioenergetics}

Astrocytes were plated on Seahorse XF-24 plates at a density of $1.5 \times 10^{4}$ cells per well and incubated in complete culture medium for two days at $37^{\circ} \mathrm{C}$ in $5 \% \mathrm{CO}_{2} .24$ hours before the experiment, cells were either infected by SARS-CoV-2 (MOI 0.1) or not infected (MOCK). One day post-infection, the culture medium was changed to Seahorse Base medium (supplemented with $1 \mathrm{mM}$ pyruvate, $2 \mathrm{mM}$ glutamine, and $10 \mathrm{mM}$ glucose) and cells were incubated at $37^{\circ} \mathrm{C}$ in a non- $\mathrm{CO}_{2}$ incubator for 1 hour. OCR (Oxygen Consumption Rate) was measured over the course of the experiment under basal conditions and after the injections of oligomycin $(1 \mu \mathrm{M})$, FCCP $(5 \mu \mathrm{M})$ and antimycin A/rotenone $(100 \mathrm{nM} / 1 \mu \mathrm{M})$. Protein concentration was determined for each well to normalize the data. Data were expressed as mean \pm SEM of at least two independent experiments performed in quintuplicate. Statistical significance analysis was 
calculated by two-tailed unpaired Student's t-test. All analyses were performed using GraphPad Prism 8.0 software (San Diego, CA, USA) and a significance level of $\mathrm{p} \leq 0.05$ was adopted.

\section{SH-SY5Y culture in astrocytes conditioned medium}

Astrocytes and SH-SY5Y cells were cultured separately in standard conditions until complete differentiation. Next, astrocytes were infected either with MOCK or SARS-CoV-2 (MOI 0.1) and after 24 hours, the medium was removed and cells were washed with PBS and cultured for 24 hours. The SH-SY5Y medium was removed and replaced by the conditioned medium of SARS-CoV-2-infected astrocytes or control medium for 24 hours. After incubation, cells and the medium were collected for Flow Cytometer Analysis, following the described procedure below.

\section{Flow cytometer analysis}

SH-SY5Y cell viability was determined $24 \mathrm{~h}$ after incubation with the conditioned medium of SARS-CoV-2-infected astrocytes or control medium. The percentage of live (Apotracker-/FVS510-), necrotic (Apotracker-/FVS510+), early (Apotracker+/FVS510-) and late (Apotracker+/FVS510+) apoptotic cells was determined by flow cytometry (FACSymphony Becton \& Dickinson, San Diego, CA, USA), after labeling with fixable viability stain (FVS510, BD Biosciences, \#564406) and ApotrackerTM Green (BioLegend, \#427403). Data were analyzed using FlowJo software (BD Biosciences). Data are representative of at least two independent experiments performed in triplicate and are shown as mean \pm SEM. P values were determined by ANOVA one-ay followed by Tukey's post hoc test. 


\section{Data availability}

The mass spectrometry proteomic data have been deposited to the ProteomeXchange Consortium via the PRIDE ${ }^{62}$ partner repository with the dataset identifier PXD021812 and 10.6019/PXD021812.

\section{Acknowledgements}

We thank Edison Durigon for providing the SARS-CoV-2. We thank Gabriela Lopes Vitória, Elzira E. Saviani and Paulo Baldasso for technical support. The authors would like to thank FAPESP (São Paulo Research Foundation; grants 2020/04746-0; 2017/25588-1; 2019/00098-7; 2014/10068-4; 2020/04919-2; 2013/08216-2; 2020/05601-6; 2020/04860-8; 2019/11457-8; 2013/07559-3; 2013/07607-8), Fundo de Apoio ao Ensino, Pesquisa e Extensão (FAEPEX) Unicamp - Grant number: 2274/20, the Brazilian National Council for Scientific and Technological Development - CNPq, and the Coordenação de Aperfeiçoamento de Pessoal de Nível Superior - Brazil (CAPES). The authors wish to thank Bradley J. Smith, MSc for English review support.

\section{Author Contributions}

F.C., V.C.C. and F.P.V. designed the majority of the experiments, performed the experiments, analyzed data and contributed to writing the manuscript. P.H.V. performed metabolomic analysis. A.G.F.V., A.S.L.M.A., C.B-T., G.S.Z., G. R.-O., L.C.a, V.M.S-C., A.C.C., contributed to the study design, performed experiments and data interpretation. F.C., A.C.C., A.G.F.V., A.S.L.M.A., C.B-T., G.S.Z., V.M.S-C., performed cell culture care and cell differentiation, flow cytometry and RT-PCRs analysis. A.S.L.M.A., A.C.C., P.L.P., D.A.T.T., 
G.F.S., S.P.M., R.G.L., J.F., M.R., N.B.S., M. C.M., R.E.M.P.S., performed SARS-CoV-2 In vitro infections and BSL-3 work. R.G.L., G.P.R., T.L.K., G.G.D., J.A.G., P.B.R., N.B.S. performed experiments and discussed the data. V.C.C., G.R.O., L.C., performed all proteomic experiments and systems biology analysis in silico. R.B.J., L.S.S., M.H.N., I.K.A., M.R.B., M.K.M.A., J.R.S.J., L.L.D., M.E.P.C.S., I.M.P.S., E.D.R., S.M.G., L.H.L.S., V.B., B.M.C., F.C. and C.L.Y. recruited patients, performed brain imaging and clinical assessments; G.L. performed statistical analysis. T.M., A.D-N., L.F.F.S., M.D., P.S. collected human postmortem brain samples, assessed clinical files and discussed the data. M.N.B., S.B., L.S., A.F. collected human postmortem brain samples and performed histological analysis. F.P.V., B.M.S.M., G.M.A., E.M.S.F., J.C.A-F., E.A., A.S., F.Q.C., T.M.C. performed immunofluorescence analysis, RT-PCR analysis, histological and morphological analysis. A.S.V., A.D., M.A.R.V., C.D.M., S.K.R., A.S.F., P.M.M.M-V., J.L.P.M., conceived experiments, data interpretation, provided material and revised the paper. M.A.M., T.M.C., D.M.S., provided the funding for the study; formulated the hypothesis, designed experiments, analyzed data and wrote the manuscript with input from all authors. D.M.S., T.M.C. conceived and supervised the study.

\section{DECLARATION OF INTERESTS}

Authors declare no competing interests

\section{References}

1. Yang, X. et al. Clinical course and outcomes of critically ill patients with SARS-CoV-2 pneumonia in Wuhan, China: a single-centered, retrospective, observational study. Lancet Respir Med 8, 475-481 (2020). 
2. Kotfis, K. et al. COVID-19: ICU delirium management during SARS-CoV-2 pandemic. Crit. Care 24, 176 (2020).

3. Mao, L. et al. Neurologic Manifestations of Hospitalized Patients With Coronavirus Disease 2019 in Wuhan, China. JAMA Neurol. 77, 683-690 (2020).

4. Varatharaj, A. et al. Neurological and neuropsychiatric complications of COVID-19 in 153 patients: a UK-wide surveillance study. Lancet Psychiatry 7, 875-882 (2020).

5. Lu, Y. et al. Cerebral Micro-Structural Changes in COVID-19 Patients - An MRI-based 3month Follow-up Study. EClinicalMedicine 25, 100484 (2020).

6. Zhou, F. et al. Clinical course and risk factors for mortality of adult inpatients with COVID19 in Wuhan, China: a retrospective cohort study. Lancet 395, 1054-1062 (2020).

7. De Felice, F. G., Tovar-Moll, F., Moll, J., Munoz, D. P. \& Ferreira, S. T. Severe Acute Respiratory Syndrome Coronavirus 2 (SARS-CoV-2) and the Central Nervous System. Trends Neurosci. 43, 355-357 (2020).

8. Xu, J. et al. Detection of Severe Acute Respiratory Syndrome Coronavirus in the Brain: Potential Role of the Chemokine Mig in Pathogenesis. Clinical Infectious Diseases vol. 41 1089-1096 (2005).

9. Lau, K.-K. et al. Possible Central Nervous System Infection by SARS Coronavirus. Emerging Infectious Diseases vol. 10 342-344 (2004).

10. Hung, E. C. W. et al. Detection of SARS Coronavirus RNA in the Cerebrospinal Fluid of a Patient with Severe Acute Respiratory Syndrome. Clinical Chemistry vol. 49 2108-2109 (2003).

11. Moriguchi, T. et al. A first case of meningitis/encephalitis associated with SARSCoronavirus-2. Int. J. Infect. Dis. 94, 55-58 (2020). 
12. Huang, Y. H., Hanna Huang, Y., Jiang, D. \& Huang, J. T. SARS-CoV-2 Detected in Cerebrospinal Fluid by PCR in a Case of COVID-19 Encephalitis. Brain, Behavior, and Immunity vol. 87149 (2020).

13. Bernard-Valnet, R. et al. Two patients with acute meningo-encephalitis concomitant to SARS-CoV-2 infection. doi:10.1101/2020.04.17.20060251.

14. Politi, L. S., Salsano, E. \& Grimaldi, M. Magnetic Resonance Imaging Alteration of the Brain in a Patient With Coronavirus Disease 2019 (COVID-19) and Anosmia. JAMA Neurol. 77, 1028-1029 (2020).

15. Reichard, R. R. et al. Neuropathology of COVID-19: a spectrum of vascular and acute disseminated encephalomyelitis (ADEM)-like pathology. Acta Neuropathologica vol. 140 $1-6(2020)$.

16. Matschke, J. et al. Neuropathology of patients with COVID-19 in Germany: a post-mortem case series. Lancet Neurol. (2020) doi:10.1016/S1474-4422(20)30308-2.

17. Song, E. et al. Neuroinvasion of SARS-CoV-2 in human and mouse brain. bioRxiv (2020) doi:10.1101/2020.06.25.169946.

18. Biega, T. J., Lonser, R. R. \& Butman, J. A. Differential cortical thickness across the central sulcus: a method for identifying the central sulcus in the presence of mass effect and vasogenic edema. AJNR Am. J. Neuroradiol. 27, 1450-1453 (2006).

19. Li, B. et al. Prevalence and impact of cardiovascular metabolic diseases on COVID-19 in China. Clin. Res. Cardiol. 109, 531-538 (2020).

20. Weber, F., Wagner, V., Rasmussen, S. B., Hartmann, R. \& Paludan, S. R. Double-stranded RNA is produced by positive-strand RNA viruses and DNA viruses but not in detectable amounts by negative-strand RNA viruses. J. Virol. 80, 5059-5064 (2006). 
21. Rouach, N., Koulakoff, A., Abudara, V., Willecke, K. \& Giaume, C. Astroglial Metabolic Networks Sustain Hippocampal Synaptic Transmission. Science vol. 322 1551-1555 (2008).

22. Liddelow, S. A. et al. Neurotoxic reactive astrocytes are induced by activated microglia. Nature 541, 481-487 (2017).

23. Yun, S. P. et al. Block of A1 astrocyte conversion by microglia is neuroprotective in models of Parkinson's disease. Nat. Med. 24, 931-938 (2018).

24. Guttenplan, K. A. et al. Knockout of reactive astrocyte activating factors slows disease progression in an ALS mouse model. Nat. Commun. 11, 3753 (2020).

25. Simpson, I. A., Carruthers, A. \& Vannucci, S. J. Supply and demand in cerebral energy metabolism: The role of nutrient transporters. J. Cereb. Blood Flow Metab. 27, 1766-1791 (2007).

26. Gandhi, G. K., Cruz, N. F., Ball, K. K. \& Dienel, G. A. Astrocytes are poised for lactate trafficking and release from activated brain and for supply of glucose to neurons. $J$. Neurochem. 111, 522-536 (2009).

27. Kovalevich, J. \& Langford, D. Considerations for the use of SH-SY5Y neuroblastoma cells in neurobiology. Methods Mol. Biol. 1078, 9-21 (2013).

28. Troyer, E. A., Kohn, J. N. \& Hong, S. Are we facing a crashing wave of neuropsychiatric sequelae of COVID-19? Neuropsychiatric symptoms and potential immunologic mechanisms. Brain Behav. Immun. 87, 34-39 (2020).

29. Rogers, J. P. et al. Psychiatric and neuropsychiatric presentations associated with severe coronavirus infections: a systematic review and meta-analysis with comparison to the COVID-19 pandemic. Lancet Psychiatry 7, 611-627 (2020). 
30. Jacob, F. et al. Human Pluripotent Stem Cell-Derived Neural Cells and Brain Organoids Reveal SARS-CoV-2 Neurotropism. doi:10.1101/2020.07.28.225151.

31. Yavarpour-Bali, H. \& Ghasemi-Kasman, M. Update on neurological manifestations of COVID-19. Life Sci. 257, 118063 (2020).

32. Turner, D. A. \& Adamson, D. C. Neuronal-astrocyte metabolic interactions: understanding the transition into abnormal astrocytoma metabolism. J. Neuropathol. Exp. Neurol. 70, 167$176(2011)$.

33. Suzuki, A. et al. Astrocyte-neuron lactate transport is required for long-term memory formation. Cell 144, 810-823 (2011).

34. Walls, A. B. et al. Knockout of GAD65 has major impact on synaptic GABA synthesized from astrocyte-derived glutamine. J. Cereb. Blood Flow Metab. 31, 494-503 (2011).

35. Bélanger, M., Allaman, I. \& Magistretti, P. J. Brain energy metabolism: focus on astrocyteneuron metabolic cooperation. Cell Metab. 14, 724-738 (2011).

36. Bak, L. K., Schousboe, A. \& Waagepetersen, H. S. The glutamate/GABA-glutamine cycle: aspects of transport, neurotransmitter homeostasis and ammonia transfer. Journal of Neurochemistry vol. 98 641-653 (2006).

37. Bonansco, C. et al. Glutamate released spontaneously from astrocytes sets the threshold for synaptic plasticity. European Journal of Neuroscience vol. 33 1483-1492 (2011).

38. Milad, M. R. \& Rauch, S. L. The role of the orbitofrontal cortex in anxiety disorders. Ann. N. Y. Acad. Sci. 1121, 546-561 (2007).

39. Knutson, K. M. et al. Areas of Brain Damage Underlying Increased Reports of Behavioral Disinhibition. J. Neuropsychiatry Clin. Neurosci. 27, 193-198 (2015).

40. Mao, X.-Y. \& Jin, W.-L. The COVID-19 Pandemic: Consideration for Brain Infection. 
Neuroscience 437, 130-131 (2020).

41. Whelan, C. D. et al. Structural brain abnormalities in the common epilepsies assessed in a worldwide ENIGMA study. Brain 141, 391-408 (2018).

42. Garcia, D. D. S. et al. Anxiety and depression symptoms disrupt resting state connectivity in patients with genetic generalized epilepsies. Epilepsia 60, 679-688 (2019).

43. Bolognani, S. A. P. et al. Development of alternative versions of the Logical Memory subtest of the WMS-R for use in Brazil. Dement. neuropsychol. 9, 136-148 (2015).

44. Campanholo, K. R. et al. Performance of an adult Brazilian sample on the Trail Making Test and Stroop Test. Dement Neuropsychol 8, 26-31 (2014).

45. Jackson, C. The Chalder Fatigue Scale (CFQ 11). Occup. Med. 65, 86 (2015).

46. Righart, R. et al. Volume versus surface-based cortical thickness measurements: A comparative study with healthy controls and multiple sclerosis patients. PLoS One 12, e0179590 (2017)

47. Genovese, C. R., Lazar, N. A. \& Nichols, T. Thresholding of statistical maps in functional neuroimaging using the false discovery rate. Neuroimage 15, 870-878 (2002).

48. Destrieux, C., Fischl, B., Dale, A. \& Halgren, E. Automatic parcellation of human cortical gyri and sulci using standard anatomical nomenclature. Neuroimage 53, 1-15 (2010).

49. Team, R. C. RA language and environment for statistical computing. Versión 3.4. 3, Vienna, Austria, R Foundation for Statistical Computing. (2017).

50. Fraga, A. M. et al. Establishment of a Brazilian Line of Human Embryonic Stem Cells in Defined Medium: Implications for Cell Therapy in an Ethnically Diverse Population. Cell Transplant. 20, 431-440 (2011).

51. Trindade, P. et al. Short and long TNF-alpha exposure recapitulates canonical astrogliosis 
events in human-induced pluripotent stem cells-derived astrocytes. Glia 68, 1396-1409 (2020).

52. Distler, U., Kuharev, J., Navarro, P. \& Tenzer, S. Label-free quantification in ion mobilityenhanced data-independent acquisition proteomics. Nat. Protoc. 11, 795-812 (2016).

53. Shannon, P. et al. Cytoscape: a software environment for integrated models of biomolecular interaction networks. Genome Res. 13, 2498-2504 (2003).

54. Yu, G., Wang, L.-G., Han, Y. \& He, Q.-Y. clusterProfiler: an R package for comparing biological themes among gene clusters. OMICS 16, 284-287 (2012).

55. Zhang, X. et al. CellMarker: a manually curated resource of cell markers in human and mouse. Nucleic Acids Res. 47, D721-D728 (2019).

56. Kanehisa, M. \& Goto, S. KEGG: kyoto encyclopedia of genes and genomes. Nucleic Acids Res. 28, 27-30 (2000).

57. Wu, G., Feng, X. \& Stein, L. A human functional protein interaction network and its application to cancer data analysis. Genome Biol. 11, R53 (2010).

58. Schrimpe-Rutledge, A. C., Codreanu, S. G., Sherrod, S. D. \& McLean, J. A. Untargeted Metabolomics Strategies-Challenges and Emerging Directions. J. Am. Soc. Mass Spectrom. 27, 1897-1905 (2016).

59. Schmittgen, T. D. \& Livak, K. J. Analyzing real-time PCR data by the comparative C(T) method. Nat. Protoc. 3, 1101-1108 (2008).

60. Won, J. et al. Development of a Laboratory-safe and Low-cost Detection Protocol for SARS-CoV-2 of the Coronavirus Disease 2019 (COVID-19). Exp. Neurobiol. 29, 107-119 (2020).

61. Codo, A. C. et al. Elevated Glucose Levels Favor SARS-CoV-2 Infection and Monocyte 
Response through a HIF-1 $\alpha /$ Glycolysis-Dependent Axis. Cell Metab. (2020) doi:10.1016/j.cmet.2020.07.007.

62. Perez-Riverol, Y. et al. The PRIDE database and related tools and resources in 2019: improving support for quantification data. Nucleic Acids Research vol. 47 D442-D450 (2019). 
a

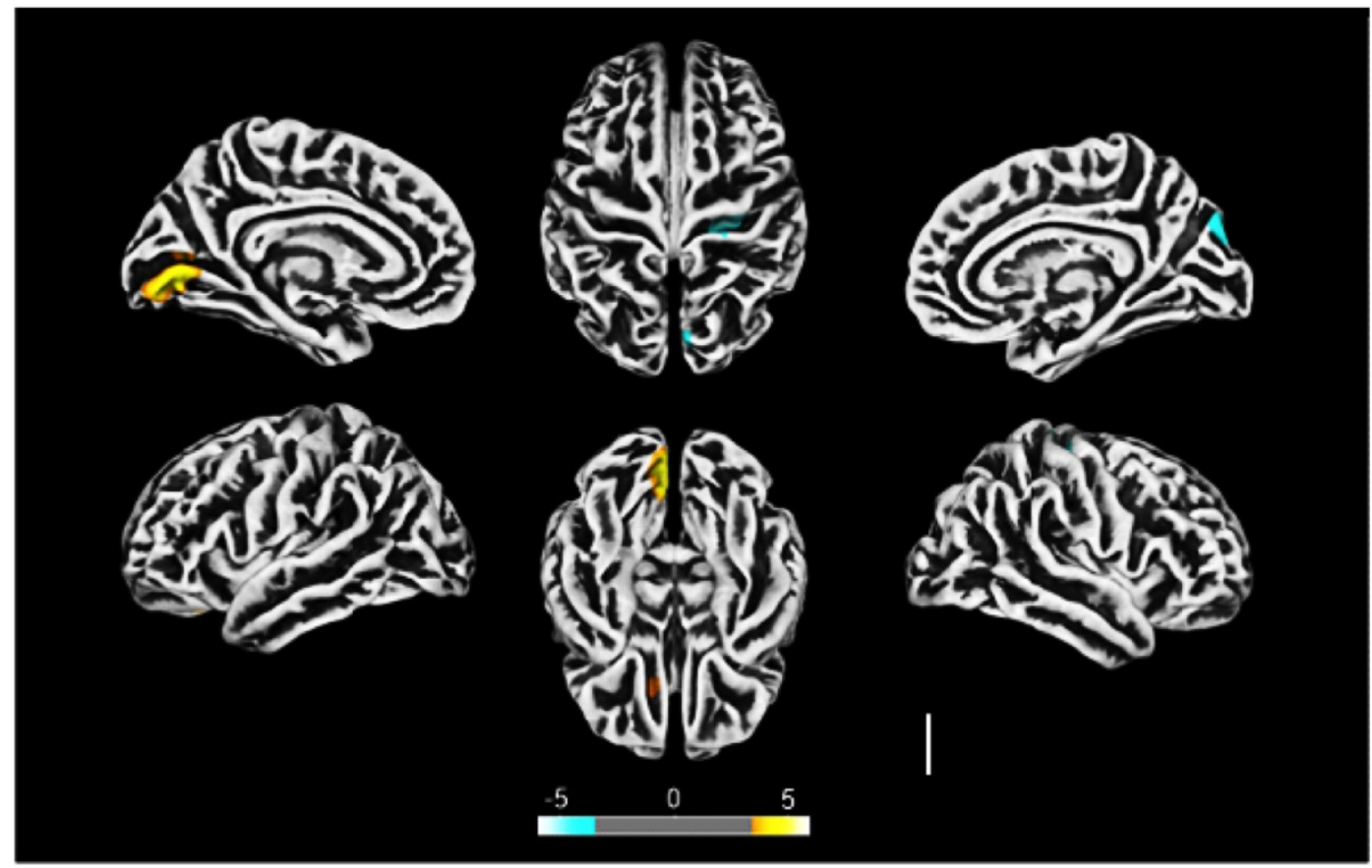

b
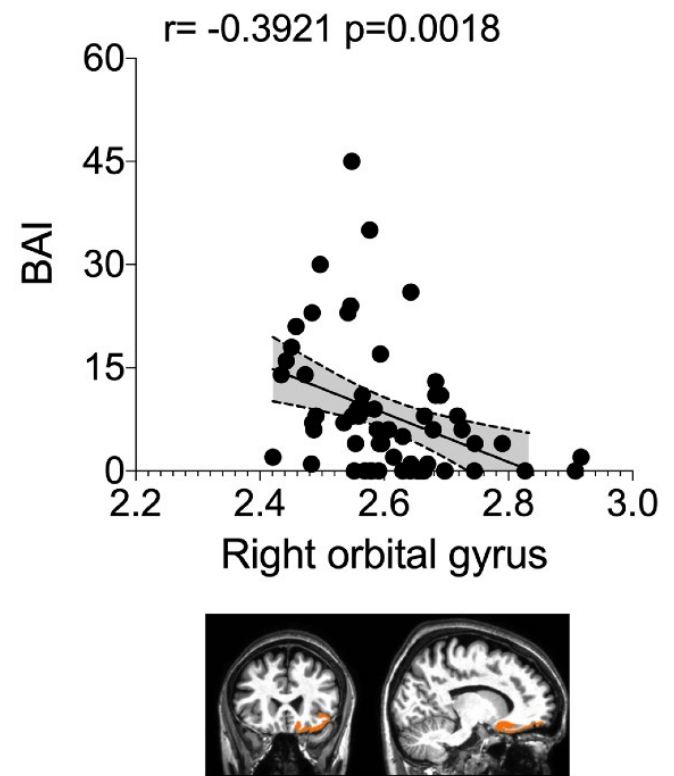
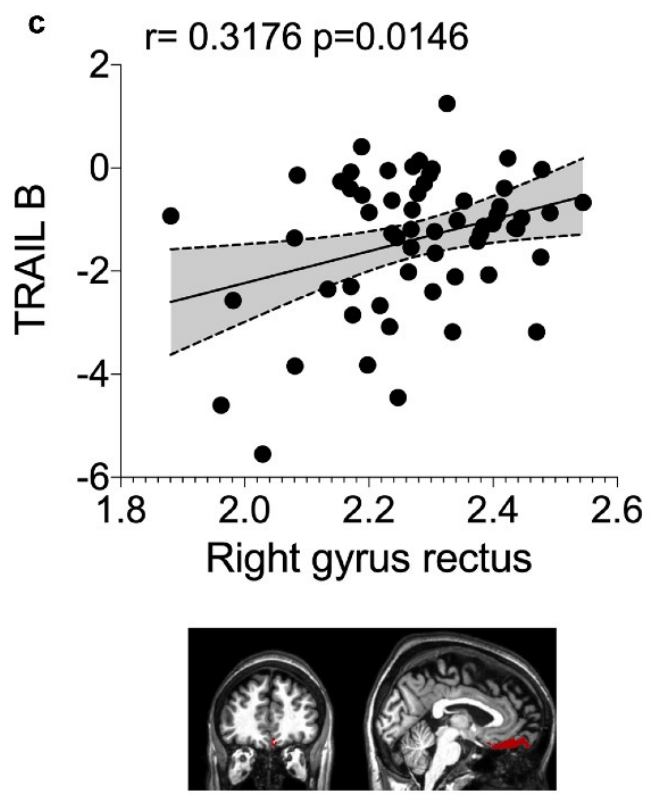

Figure 1: Altered cerebral cortical thickness is associated with neuropsychiatric symptoms in COVID-19 patients. a) Surface-based morphometry using high-resolution 3T MRI. Yellow represents areas of decreased cortical thickness: left lingual gyrus, calcarine sulcus (and cuneus), 
and olfactory sulcus (and rectus gyrus). Blue represents areas of increased cortical thickness: central sulcus (precentral and postcentral gyrus) and superior occipital gyrus. Representative image of the analysis of 81 subjects tested positive for SARS-CoV-2 (who had mild respiratory symptoms and did not require hospitalization or oxygen support) compared to 145 healthy volunteers (without diagnosis of COVID-19). The analysis was performed within a median interval of 54 days. b) Correlation between anxiety scores (BAI) and right orbital gyrus thickness. The data depicts Pearson's correlation coefficient. c) Correlation between TRAIL B performance scores and right gyrus rectus thickness. Data depict Pearson's correlation coefficient and region of interest in representative images. 
a

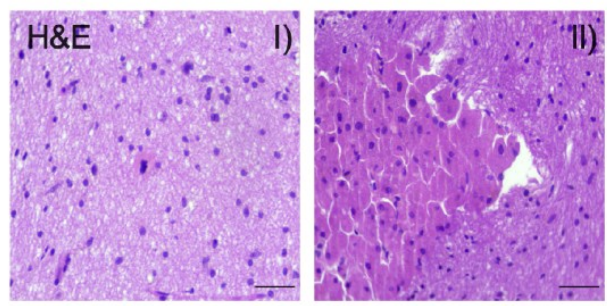

C
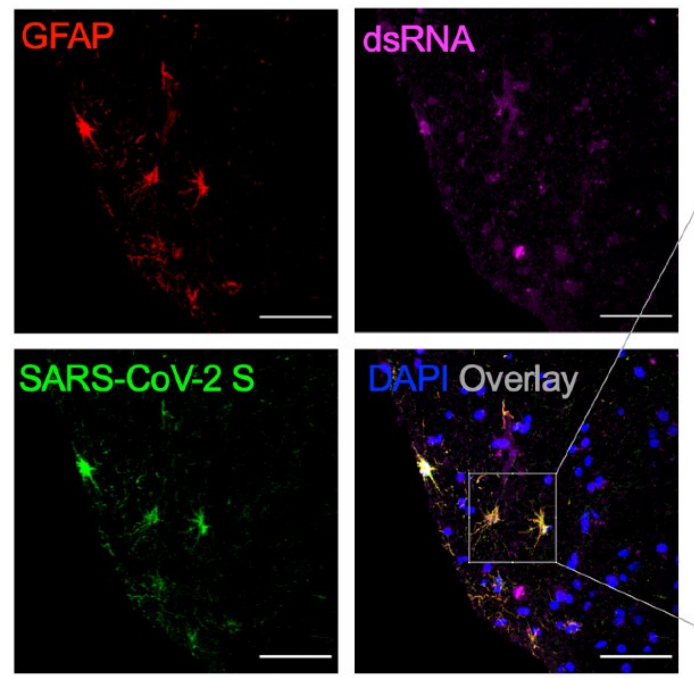

d Total of cells

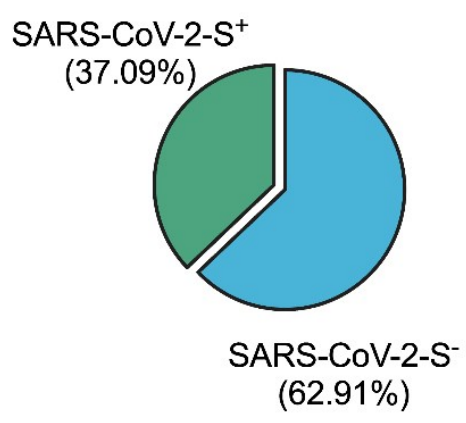

e
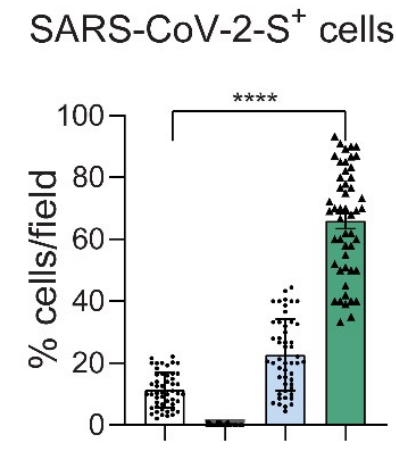

$(62.91 \%)$
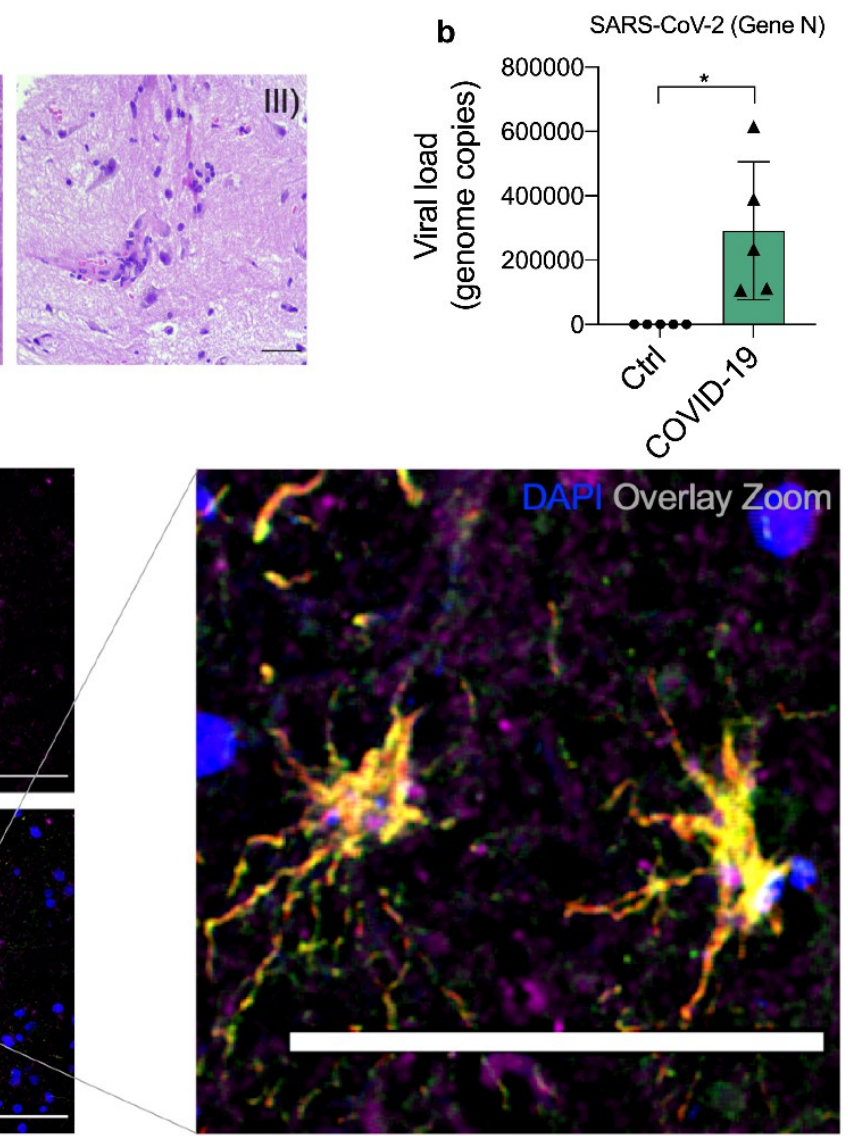

f

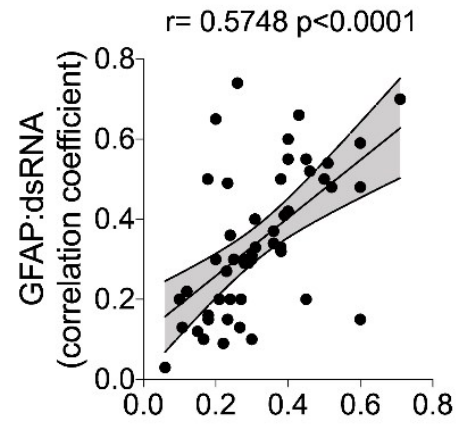

GFAP:SARS-CoV-2-S1 (correlation coefficient)

\section{Figure 2: SARS-CoV-2 infects the central nervous system, replicates in astrocytes and}

causes brain damage. a) Histopathological H\&E images of postmortem brain tissue from individuals who died of COVID-19. Five out of 26 individuals showed signs of brain damage as 
represented in the images by I) areas of necrosis, cytopathic damage (I, i.e., enlarged, hyperchromatic, atypical-appearing nuclei), vessels with margination of leukocytes and thrombus (II), and immune cells infiltrate (III). Images were acquired with 400x magnification. Scale bar indicates $50 \mu \mathrm{m}$. b) Viral load in brain tissues from the five COVID-19 patients who manifested histopathological alteration in the brain as compared to samples from SARS-CoV-2negative controls ( $\mathrm{n}=5$ per group). c) Representative confocal images of the brain tissue of one COVID-19 patient who manifested histopathological alterations. Immunofluorescence targeting glial fibrillary acidic protein (GFAP, red), double-stranded RNA (dsRNA, magenta), SARSCoV-2-S (green), and nuclei (DAPI, blue). Images were acquired with 630x magnification. Scale bar indicates $50 \mu \mathrm{m}$. d) Percentage of SARS-CoV-2-S positive cells in this tissue. e) Percentage of GFAP-positive vs. Unidentified cells, Iba1 and NeuN among infected cells. Ten fields/cases were analyzed. f) Pearson's correlation coefficient demonstrating colocalization of SARS-CoV2-S and dsRNA within GFAP-positive cells. Data shown as mean \pm SEM. P-values were determined by two-tailed unpaired tests with Welch's correction (b) or ANOVA one-way followed by Tukey's post hoc test (e). ${ }^{*} \mathrm{P}<0.05$ compared to the Ctrl-group; ${ }^{* * * *} \mathrm{P}<0.0001$ compared to Unidentified cells, Iba1 and NeuN groups. 
a
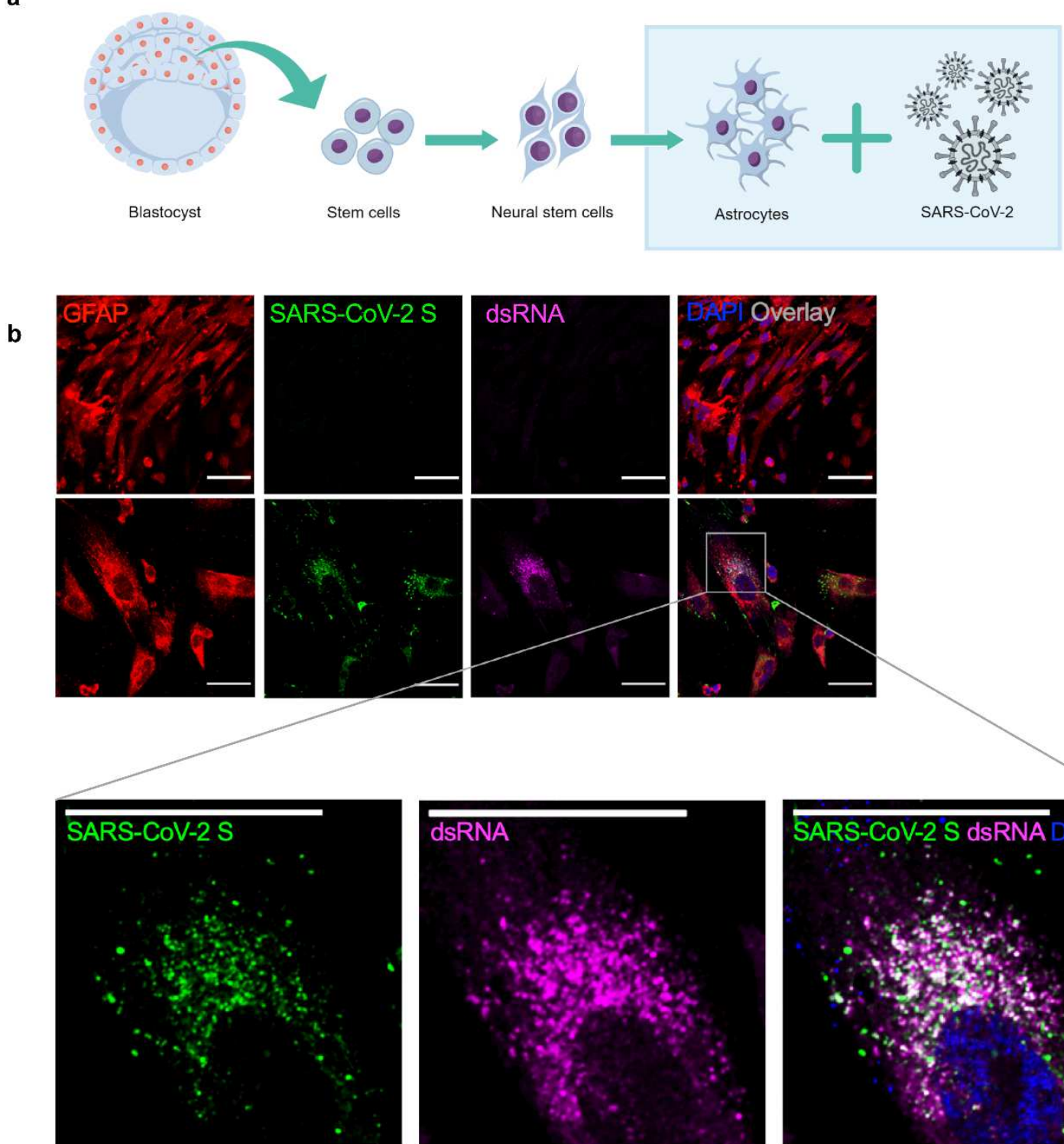

C

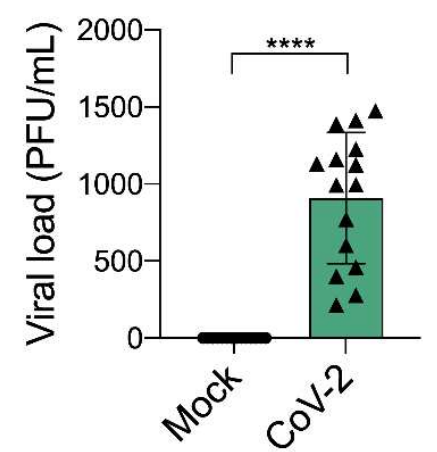

d Astrocytes

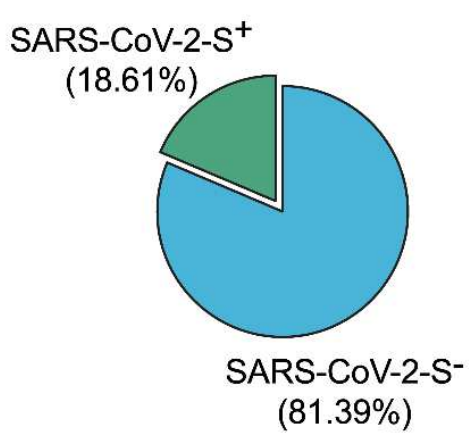

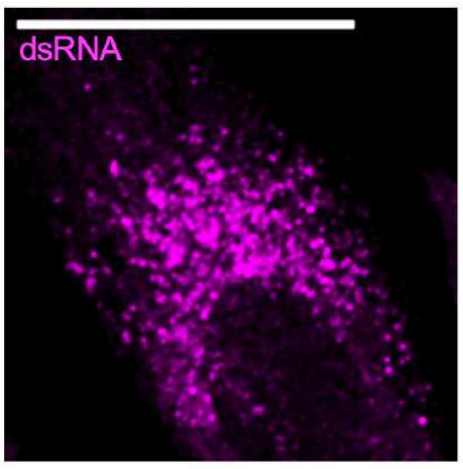

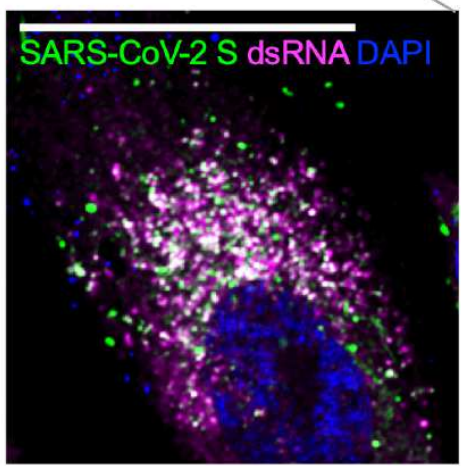

e SARS-Cov-2-S cells

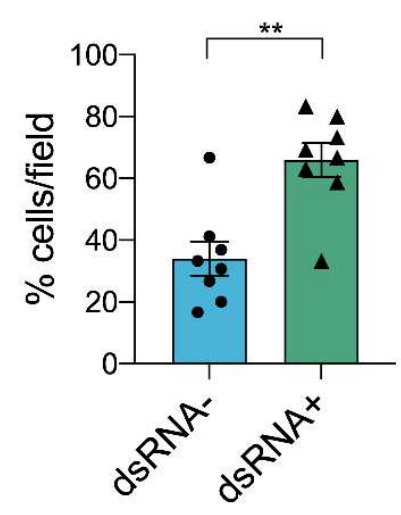


Figure 3: SARS-CoV-2 infects and replicates in astrocytes in vitro. a) Human neural stem cell-derived astrocytes were infected in vitro with SARS-CoV-2 (MOI 1) for $1 \mathrm{~h}$, washed, and harvested $24 \mathrm{~h}$ after infection. b) Immunostaining for GFAP (red), double-stranded RNA (dsRNA, magenta), SARS-CoV-2-S (green), and nuclei (DAPI, blue). Images were acquired with 630x magnification. Scale bar indicates $50 \mu \mathrm{m}$. c) SARS-CoV-2 viral load detection in astrocyte cell pellet ( $\mathrm{n}=15$ replicates) using RT-PCR. $\mathrm{d}$ ) Percentage of infected astrocytes. The data depicts SARS-CoV-2-S and DAPI stained cells (100 fields were analyzed). e) Frequency of cells containing replicating viruses. The data represents the percentage of dsRNA-stained cells into SARS-CoV-2-S positive cells (10 fields were analyzed). Data are representative of at least two independent experiments and shown as mean \pm SEM. P-values were determined by twotailed unpaired tests with Welch's correction ( $\mathrm{b}$ and $\mathrm{d}$ ). $* * \mathrm{P}<0.01 ; * * * * \mathrm{P}<0.0001$ compared to the mock group. 

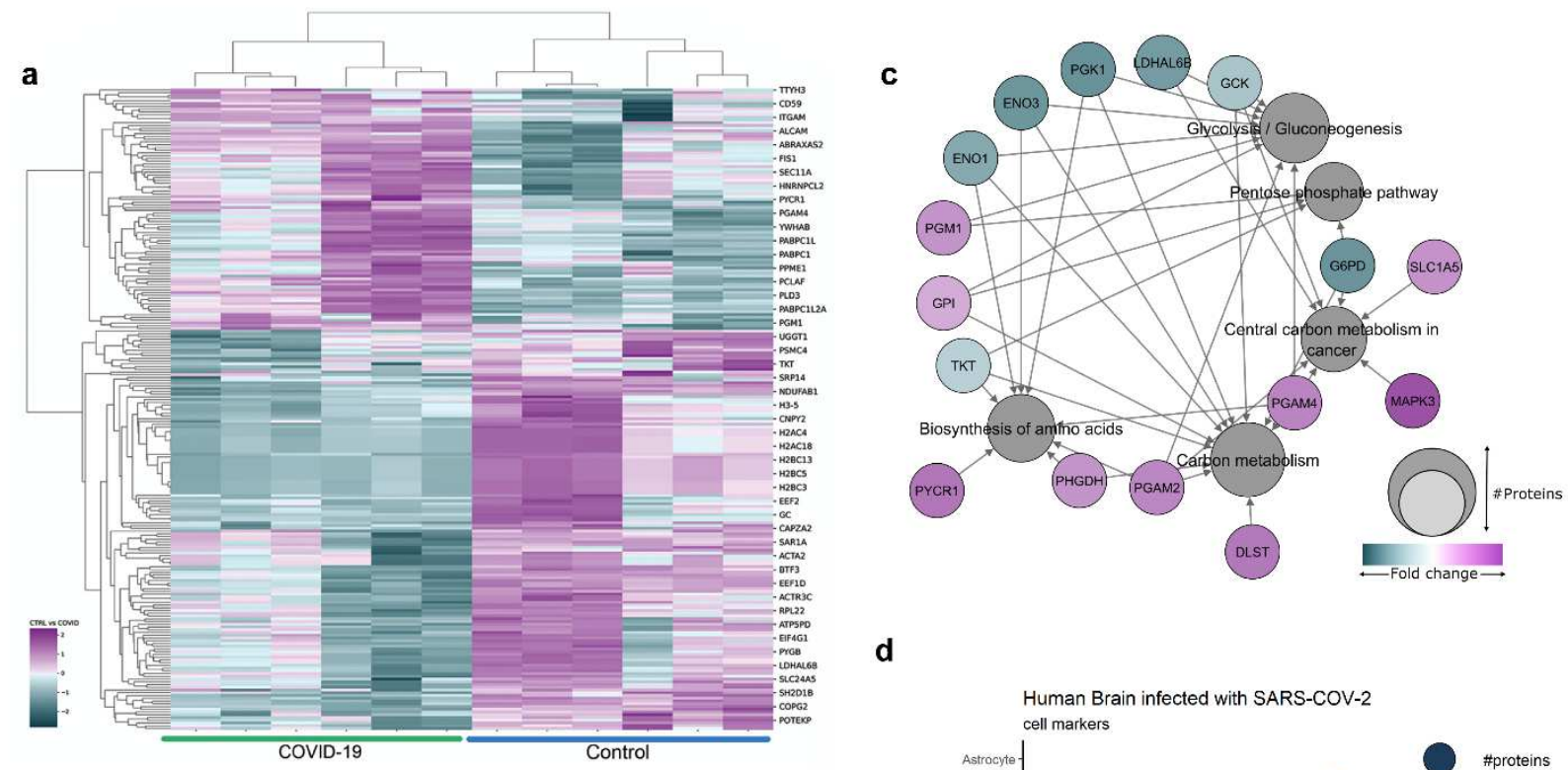

b
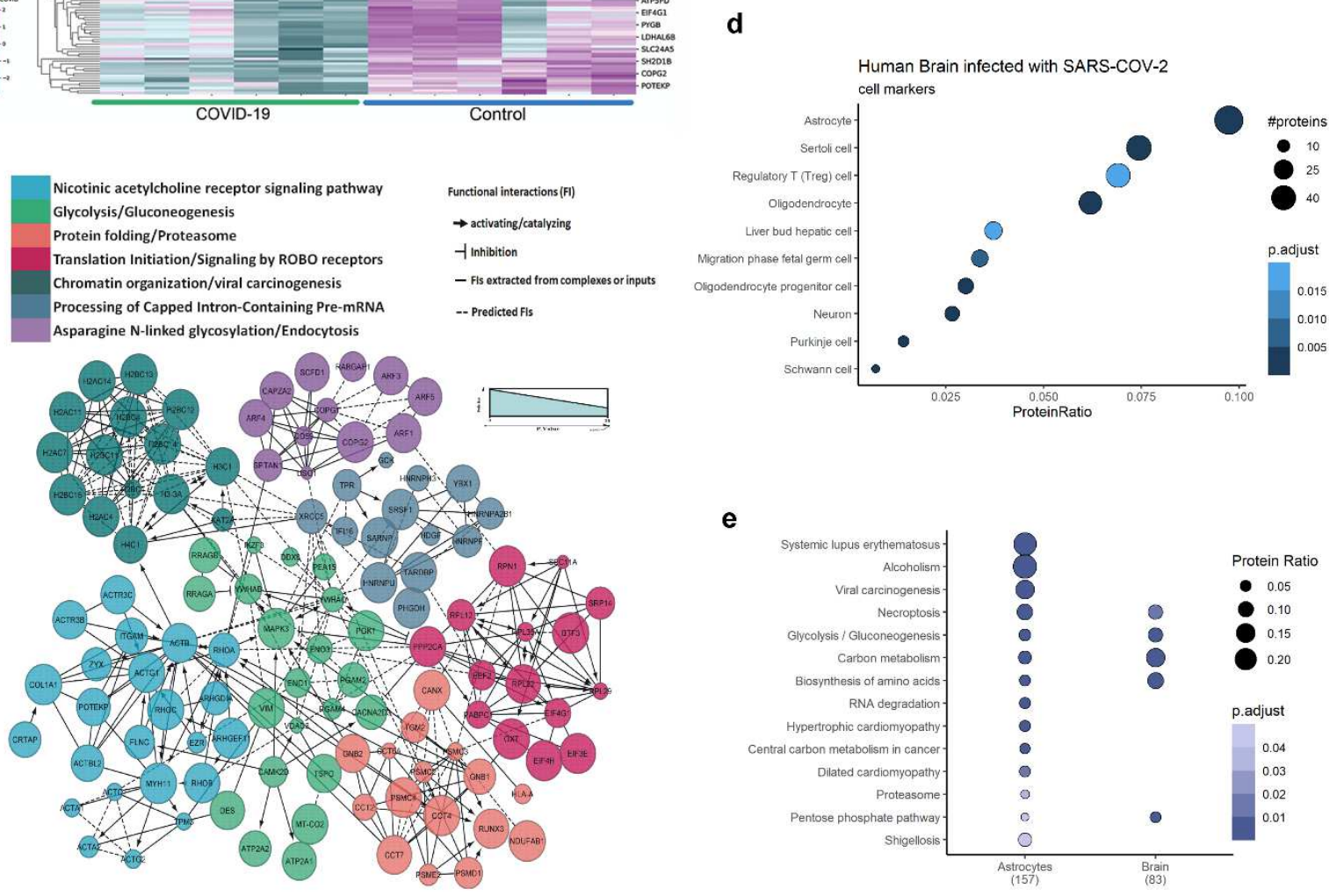

Figure 4: Proteomic changes in SARS-CoV-2-infected human astrocytes and postmortem

brain tissue from COVID-19 patients a) Hierarchical clustering of differentially expressed

proteins of human neural stem cell-derived astrocytes were infected in vitro with SARS-CoV-2

(MOI 0.1) for $1 \mathrm{~h}$, washed thoroughly and harvested after $24 \mathrm{~h}$. Mock was used as a control. b)

Reactome functional interaction network of differentially regulated genes of human neural stem 
cell-derived astrocytes were infected in vitro with SARS-CoV-2. Seven different colors show 7 protein clusters of enriched pathways specified by color and the arrows represents the proteinprotein interaction and the downstream activation or inhibition related with the gene modulation, showing how some pathways can be affected by SARS-CoV-2 infection $(\mathrm{p}<0.05$ calculated based on binomial test). c) Network of proteins found differentially regulated in SARS-COV-2infected Astrocytes and their respective pathways, enriched according to KEGG database. The pathways are represented by gray circles and their size is proportional to the number of proteins differentially regulated; proteins are represented as the smallest circles, colored according to their fold change. d) Cell type-enrichment analysis using the dataset generated by postmortem brain tissue from patients who died of Covid-19. Dot size represents the number of proteins related to the respective cell type and the $\mathrm{p}$ value adjusted by the false discovery rate (FDR). e) KEGG-enrichment analysis of differentially expressed proteins in SARS-CoV-2 infected astrocytes vs. mock as compared to postmortem brain tissue from COVID-19 patients vs. controls. Dot size represents the number of proteins related to the respective cell type and the $\mathrm{p}$ value adjusted by the false discovery rate (FDR). 

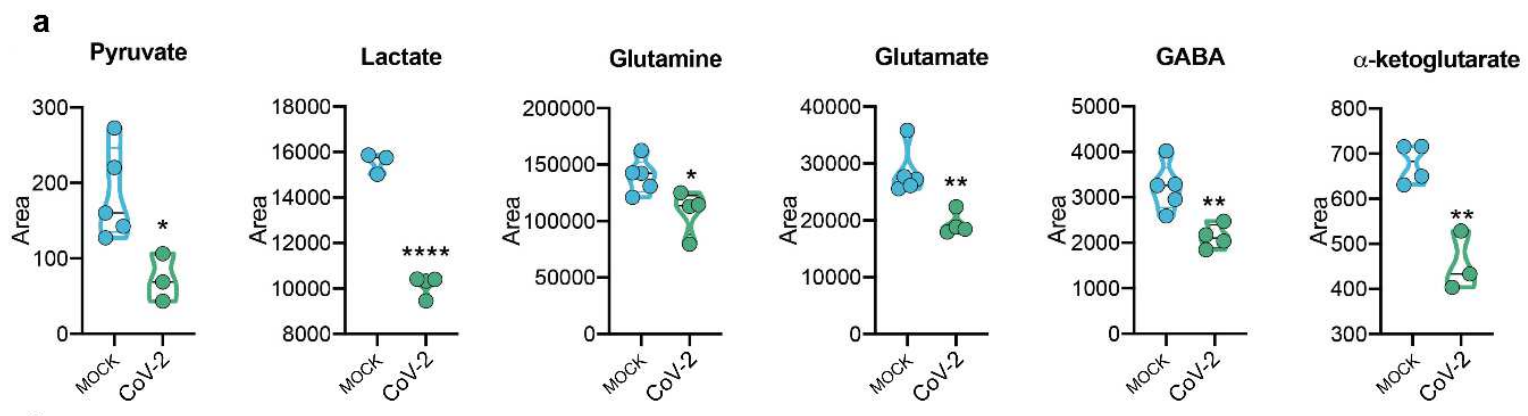

b

c
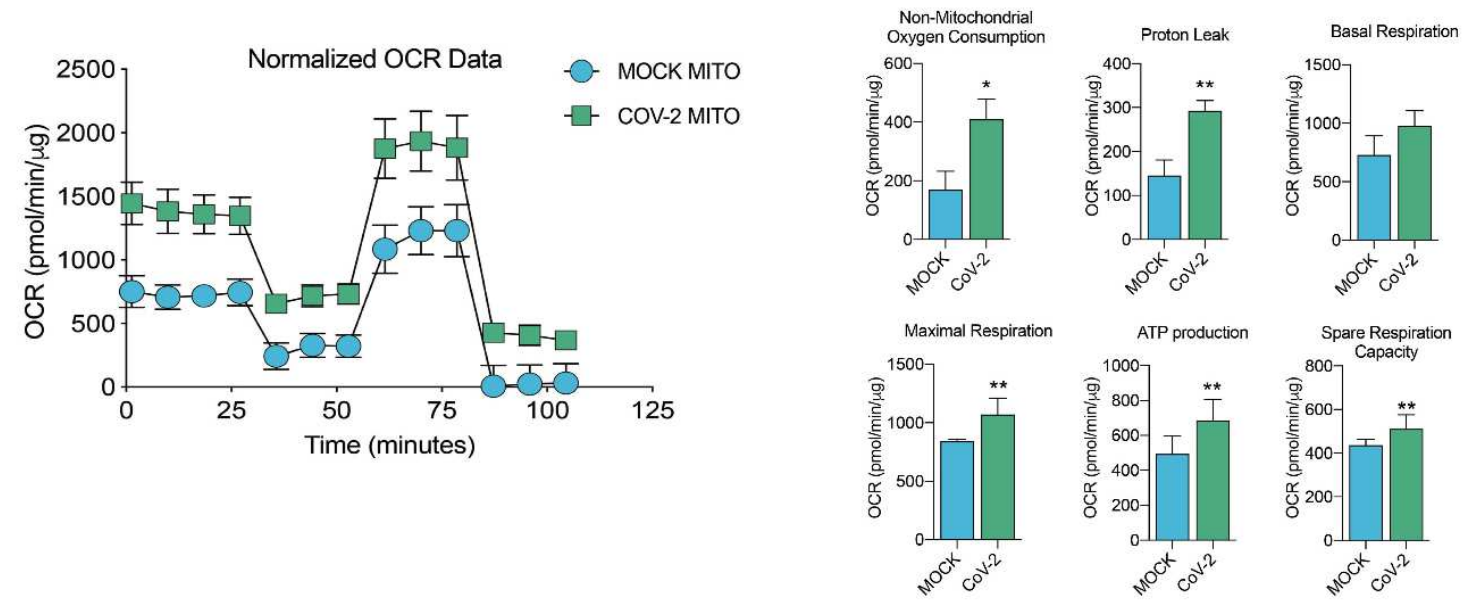

Figure 5: SARS-CoV-2 infection alters astrocyte metabolism. Human neural stem cellderived astrocytes were infected in vitro with SARS-CoV-2 (MOI 0.1) for $1 \mathrm{~h}$, washed thoroughly and harvested after 24 h. Mock was used as a control. a) High-resolution mass spectrometry quantification of pyruvate, lactate, glutamine, glutamate, GABA, and aketoglutarate in SARS-CoV-2 infected astrocytes vs. mock. The integration area of each peak was used to calculate the violin plot graph. b) Oxygen consumption rate (OCR) of SARS-CoV-2 infected astrocytes vs. mock. SeaHorse Flux Analysis using the MitoStress test where basal respiration was measured followed by determination of oligomycin-, FCCP-, and rotenone/antimycin-induced respiration. Data are representative of at least two independent experiments performed in triplicate (metabolomics analysis) or quintuplicate (SeaHorse Flux Analysis) and shown as mean \pm SEM. P values were determined by two-tailed unpaired with Welch's correction. $* \mathrm{P}<0.05 ; * * \mathrm{P}<0.01 ; * * * * \mathrm{P}<0.0001$ compared to mock. 
a

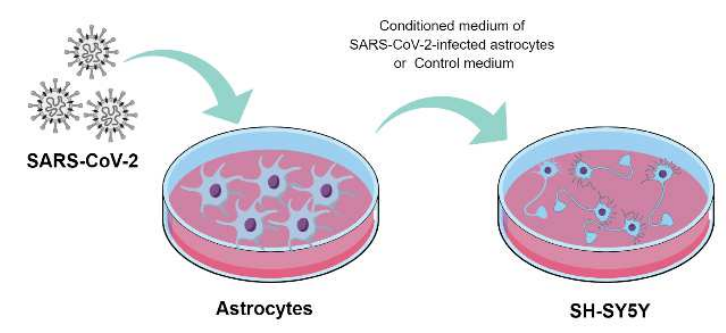

b

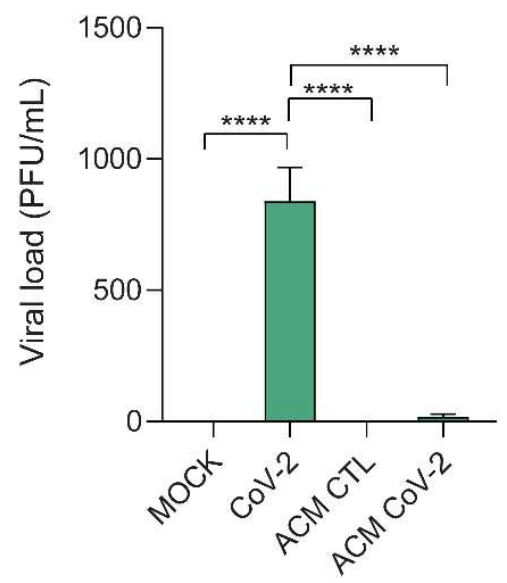

C

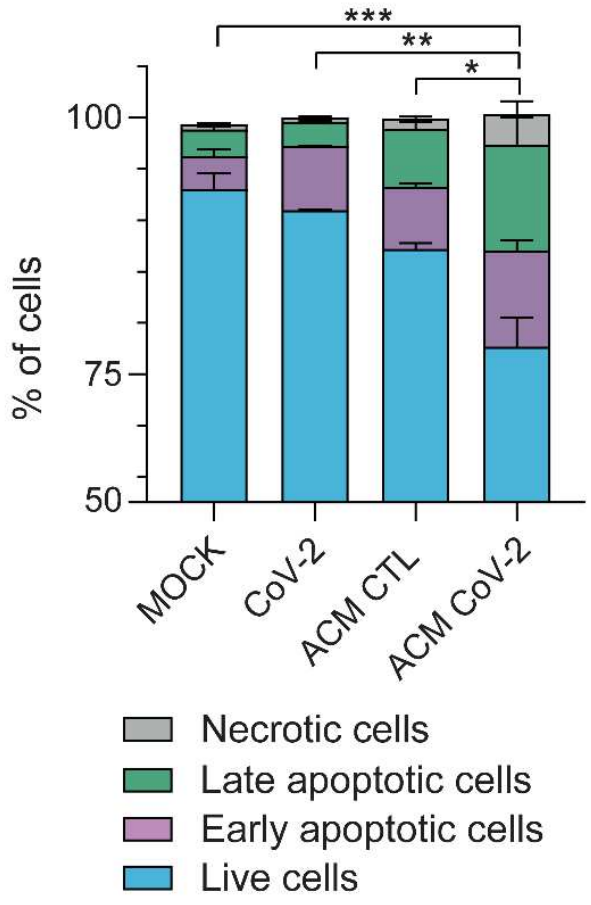

Figure 6: Conditioned medium of SARS-CoV-2-infected astrocytes reduces neuronal viability. a) Human neuronal cell line differentiated SH-SY5Y was cultured for $24 \mathrm{~h}$ in the presence of the conditioned medium of SARS-CoV-2-infected astrocytes from mock (ACM CTL) or SARS-CoV-2 (ACM CoV-2) infected cells. a) SARS-CoV-2 viral load detection in differentiated SH-SY5Y neurons cell pellet using RT-PCR. b) Cellular viability of SH-SY5Y incubated for 24 hours with conditioned medium of SARS-CoV-2-infected astrocytes (ACM) from mock (ACM CTL) or SARS-CoV-2 (ACM CoV-2) infected cells by apotracker/fixable viability stain (FVS) staining. Live (dark blue) represents double-negative population, early apoptosis (purple) and late apoptosis (green) show apotracker+FVS- and double-positive cells, respectively, and necrosis (gray) is apotracker-FVS+. Data are representative of at least two independent experiments performed in triplicate and shown as mean \pm SEM. P values were determined by one-way ANOVA followed by Tukey’s post. 


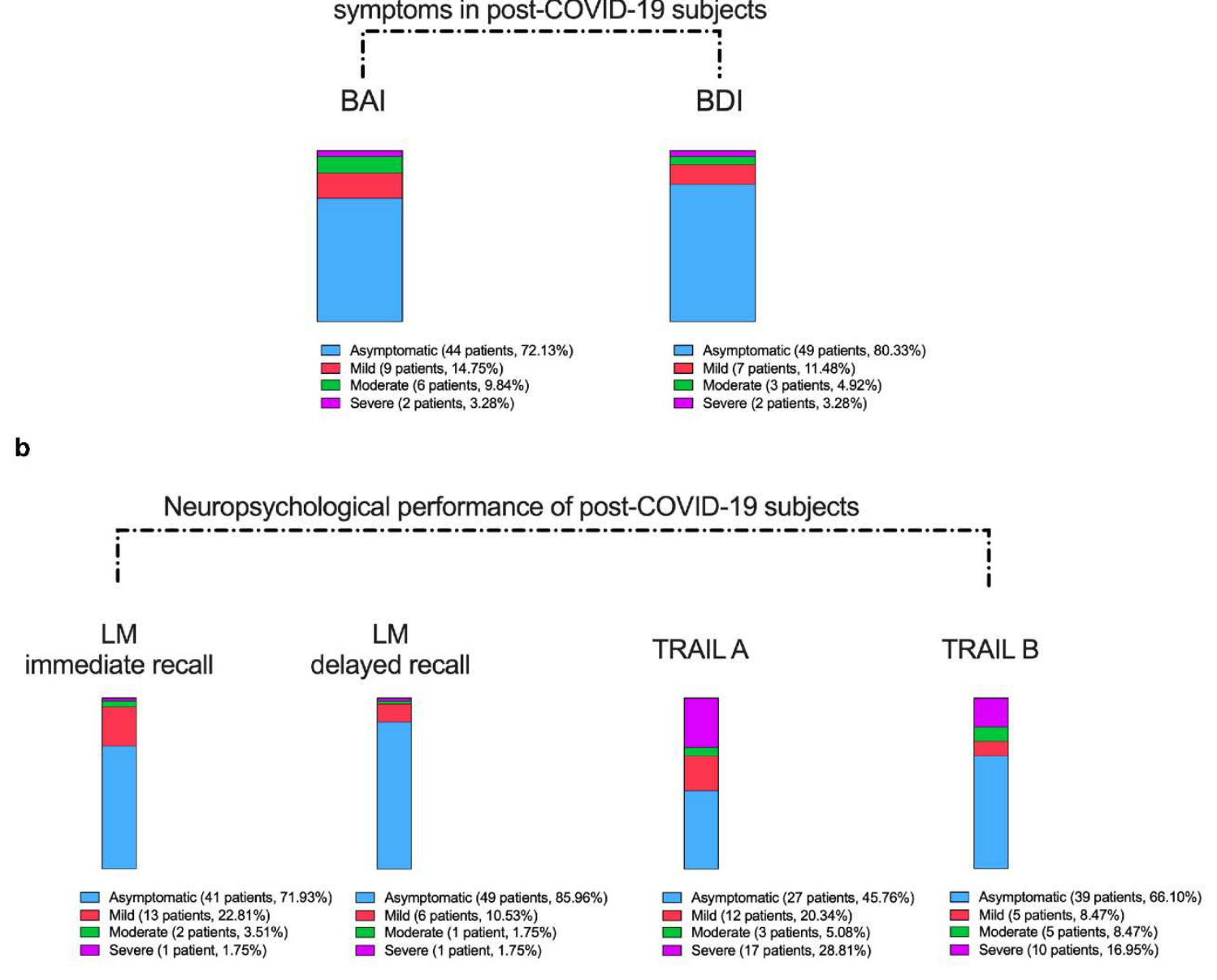

Extended data Fig. 1: Frequency of individuals with neuropsychological impairment after

mild infection. The subgroup of individuals who performed neuropsychological evaluation presented a median age of 37.8 years (range 21-63 years), 16 years of education (range 6-24 years), and 59 days of the interval from their diagnosis of COVID-19 (range 21-120 days). The median values of BDI (range $0-36)$, and also of BAI (0-45) were 6 points. The median score of CFQ-11 was 16 (range 0-32). a) Presence of anxiety (BAI) and depression (BDI) symptoms. b) Performance on the logical memory (LM) test (Wechsler Memory Scale) and cognitive function tests (TRAIL Making Test A and B). 
a
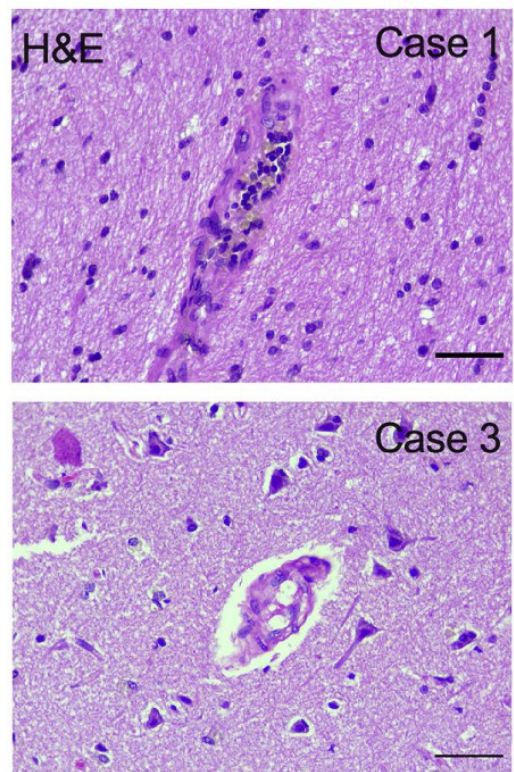

b
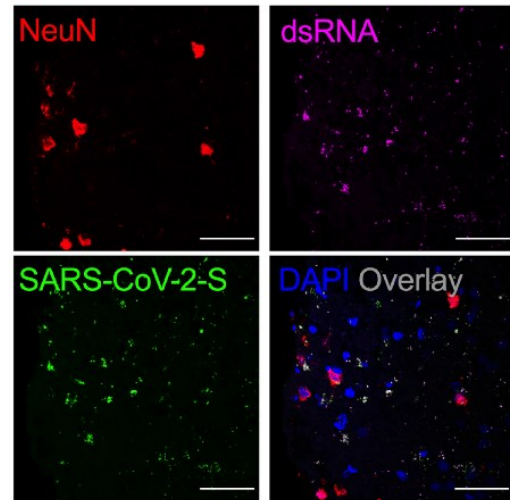

c
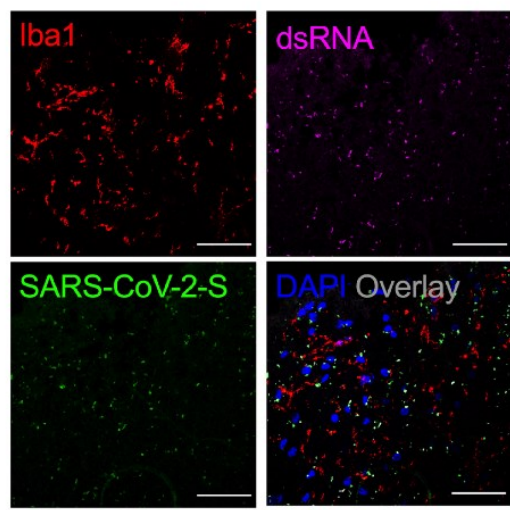
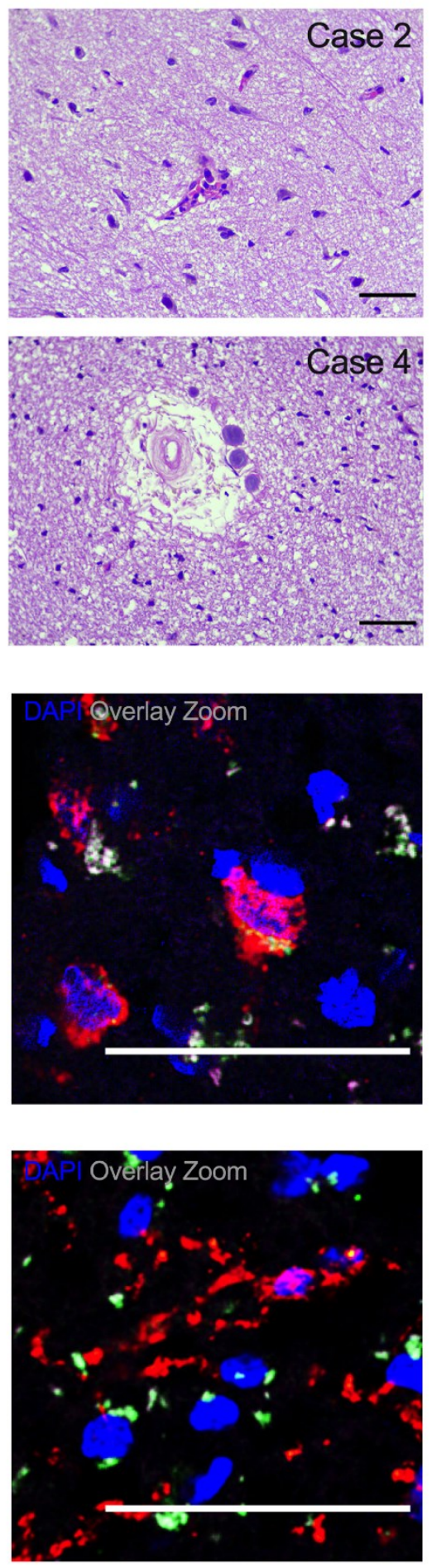

Extended data Fig. 2: SARS-CoV-2 infects neurons, but not microglia in the brain from

COVID-19. a) Histopathological alterations revealed by H\&E images of postmortem brain tissue 
from individuals who died of COVID-19. Samples from 26 individuals were analyzed and 5 showed alterations. Case 1: intraparenchymal cerebral vessel with margination of inflammatory cells through endothelium; Case 2: focal infiltration of inflammatory cells - diapedesis; Case 3: intraparenchymal vascular damage; Case 4: perivascular edema and senile corpora amylacea. bc) Representative immunostaining and confocal analysis from brain slices from autopsies of COVID-19 patients ( $\mathrm{n}=5$ ). The image depicts staining for: $\mathrm{b}$ ) nuclei (DAPI, blue), NeuN (red, neuron marker), dsRNA (magenta), and SARS-CoV-2-S (green); and c) nuclei (DAPI, blue), ionized calcium-binding adaptor molecule 1 (Iba1, red, microglia marker), dsRNA (magenta), and SARS-CoV-2-S (green). Images were acquired with 630x magnification. Scale bar indicates $50 \mu \mathrm{m}$. 


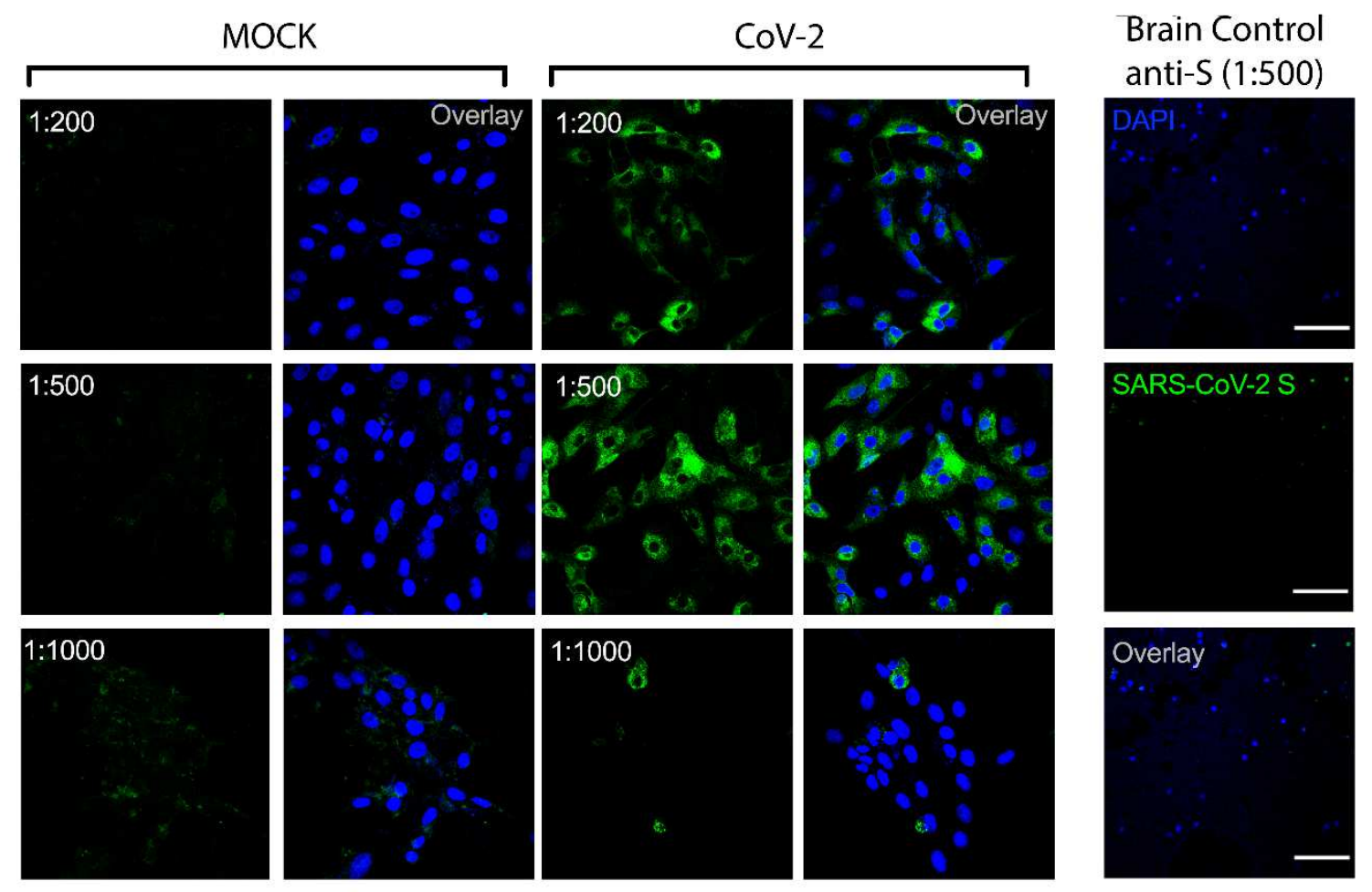

DAPI SARS-CoV-2 S (Host: human)

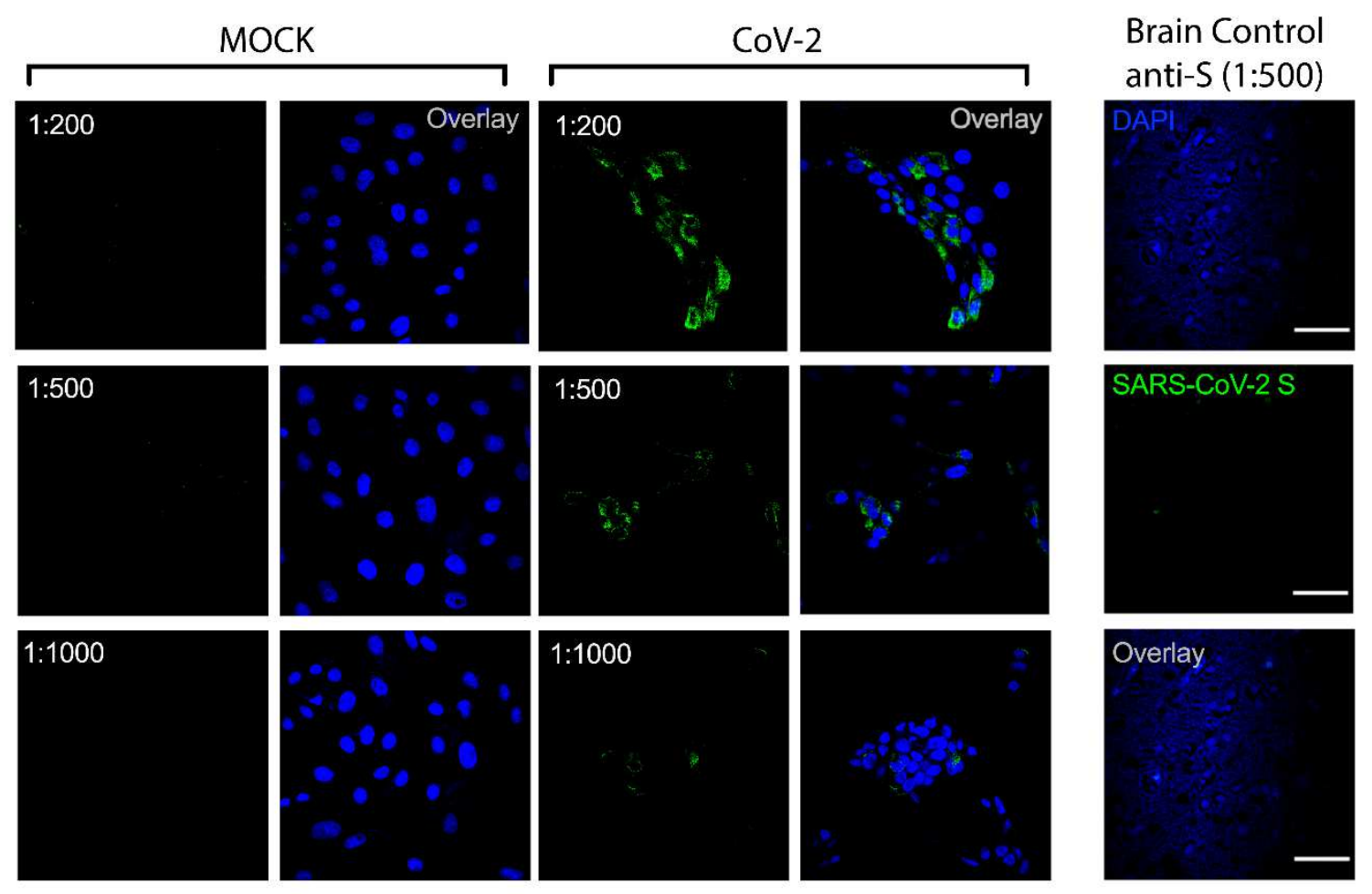

DAPI SARS-CoV-2 S (Host: rabbit) 
Extended data Fig. 3: Validation of antibodies against SARS-CoV-2 spike (S) protein.

Representative immunostaining and confocal analysis in SARS-CoV-2 infected Vero cells and brain slices from control cases (Non-COVID-19) staining with different titrations for anti-S antibodies (1:200, 1:500 and 1:1,000). Immunofluorescence images show: a) nuclei (DAPI, blue), SARS-CoV-2-S (green, human chimeric monoclonal anti-SARS-CoV-2 Spike S1, GeneScript, clone HC2001, cat. A02038) and b) nuclei (DAPI, blue), SARS-CoV-2-S (green, rabbit polyclonal anti-SARS-CoV-2 Spike, Rhea Biotech, cat. IM-0828). Images were acquired with 630x magnification at the same laser intensity. Scale bar indicates $50 \mu \mathrm{m}$. 
a CTRL vs COVID

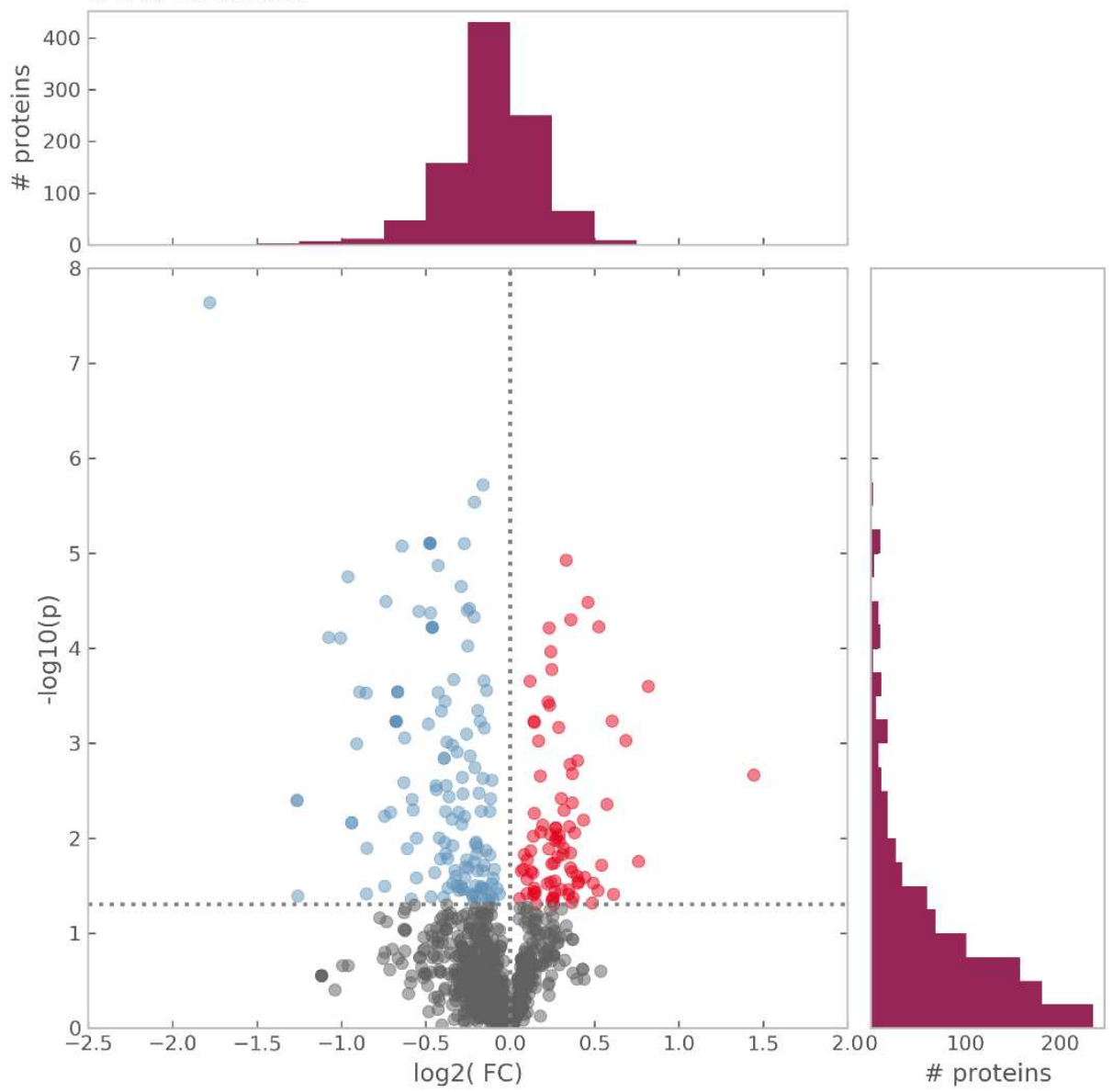

b

Pathways dysregulated in COVID

Database: KEGG

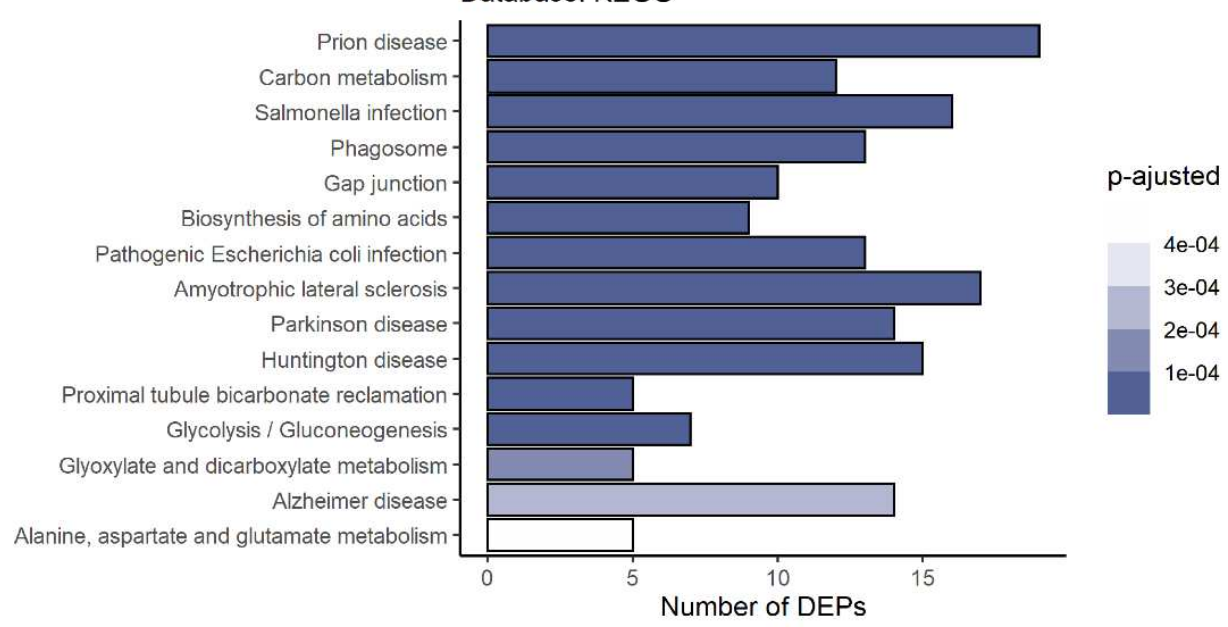

Extended data Fig. 4: Proteomic analysis of SARS-CoV-2-infected astrocytes and postmortem brain tissue from patients who died with COVID-19. a) Volcano plot representing all the 
differently regulated proteins found in Astrocytes after SARS-CoV-2 infection. b) KEGG-

enrichment analysis of differentially expressed proteins in postmortem brain tissue from patients who died with COVID-19. Dot size represents the number of proteins related to the respective cell type and the $\mathrm{p}$ value adjusted by the false discovery rate (FDR). 

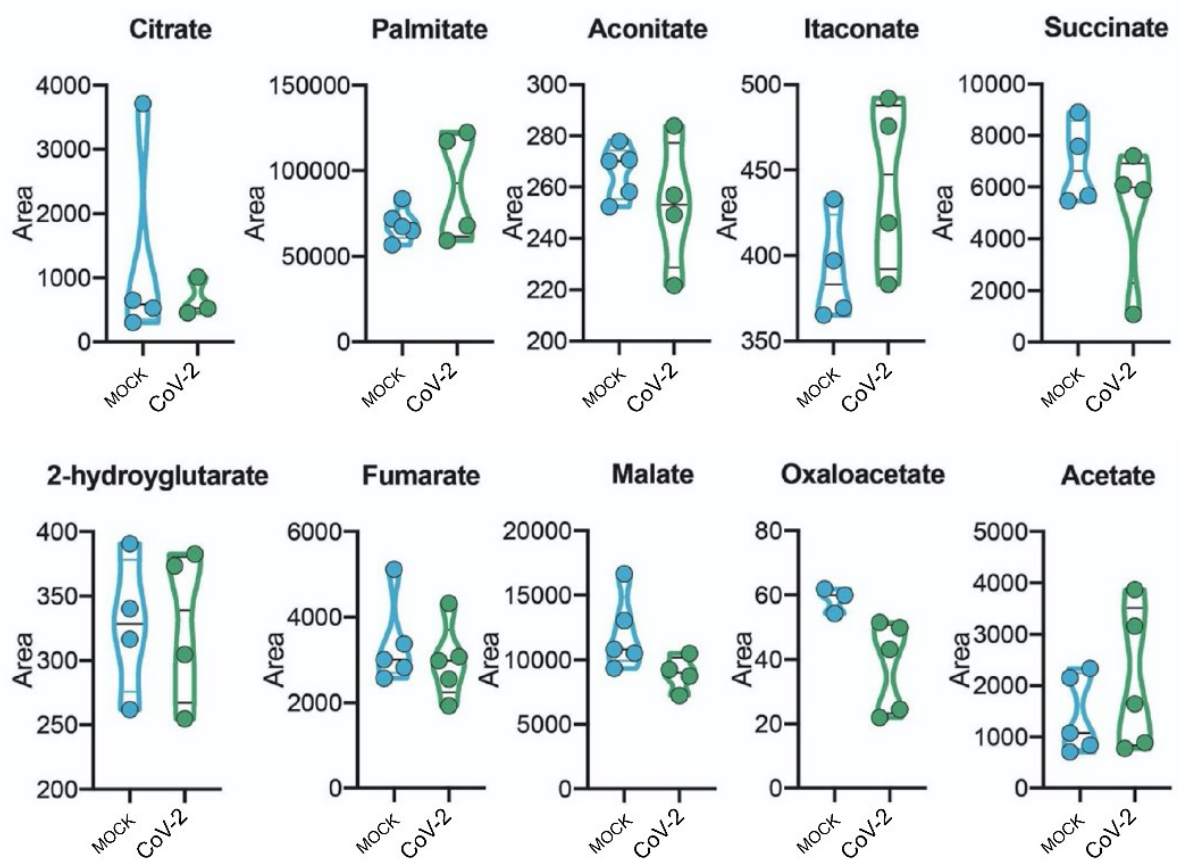

Supplementary Fig. 5: Metabolomic analysis of SARS-CoV-2-infected astrocytes. Human neural stem cell-derived astrocytes were infected in vitro with SARS-CoV-2 (MOI 0.1) for $1 \mathrm{~h}$, washed thoroughly and harvested after $24 \mathrm{~h}$. Mock was used as a control. High-resolution mass spectrometry quantification of citrate, palmitate, acetate, fumarate, succinate, oxaloacetate, and malate in SARS-CoV-2 infected astrocytes vs. mock. The integration area of each peak was used to calculate the violin plot graph and unpaired t-test with Welch's correction was used for statistical comparison. 


\begin{tabular}{cccc|}
\hline \multicolumn{2}{l}{ Table 1. Reduced cortical thickness in the left hemisphere } & \\
\hline Value (T statistic) & $\begin{array}{c}\text { Cluster size } \\
\text { (number of voxels) }\end{array}$ & Overlap of atlas region & Anatomical correspondence \\
\hline 5 & 193 & G_oc-temp_med-Lingual & Lingual gyrus \\
4.6 & 77 & S_calcarine & Calcarine sulcus \\
& G_cuneus & Cuneus \\
4 & 26 & S_parieto_occipital & Parieto-occipital sulcus \\
& & S_orbital_med-olfact & Olfactory sulcus \\
& & G_rectus & Rectus gyrus
\end{tabular}

\begin{tabular}{cccc}
\hline Table 2. Increased cortical thickness in the right hemisphere & \\
\hline Value (T statistic) & $\begin{array}{c}\text { Cluster size } \\
\text { (number of voxels) }\end{array}$ & Overlap of atlas region & Anatomical correspondence \\
\hline 5.4 & 277 & S_central & Central sulcus \\
& & G_precentral & Precentral gyrus \\
4.2 & 40 & G_postcentral & Postcentral gyrus \\
& G_occipital_sup & Superior occipital gyrus \\
\hline
\end{tabular}

\section{Table 3. Results of Neuropsychological tests (in z-score)}

\begin{tabular}{ccc}
\hline & Median & Range \\
\hline $\begin{array}{c}\text { Logical memory } \\
\text { (immediate recall) }\end{array}$ & -0.63 & -2.25 to 1.96 \\
\hline $\begin{array}{c}\text { Logical memory } \\
\text { (delayed recall) }\end{array}$ & -0.27 & -2.37 to 1.58 \\
\hline TRAIL A & -0.7 & -7 to 0.77 \\
TRAIL B & -1.13 & -5.55 to 1.25 \\
\hline
\end{tabular}


Table 4. Correlations between BAI scores and cortical thickness of orbitofrontal regions

\begin{tabular}{lccc}
\hline & Correlation & Significance & FDR adjusted significance \\
\hline Left gyrus orbital & -0.19 & 0.2 \\
\hline Right gyrus orbital & -0.42 & 0.004 \\
\hline Left gyrus rectus & -0.017 & 0.91 \\
\hline Right gyrus rectus & -0.1 & 0.5 \\
\hline FDR: false discovery rate & 0.016 \\
\hline
\end{tabular}

Table 5. Correlations between TRAIL B scores and thickness of gyrus rectus

\begin{tabular}{lccc}
\hline & Correlation & Significance & FDR adjusted significance \\
\hline Left gyrus rectus & 0.1 & 0.43 & 0.43 \\
\hline Right gyrus rectus & 0.32 & 0.014 & 0.03 \\
\hline FDR: false discovery rate & & & \\
\hline
\end{tabular}

Table 6. Correlations between Logical memory (immediate recall) scores and cortical thickness of IFG and STG.

\begin{tabular}{lccc}
\hline & Correlation & Significance & FDR adjusted significance \\
& & & 0.08 \\
\hline Left IFG (pars opercularis) & 0.27 & 0.07 & 0.1 \\
\hline Right IFG (pars opercularis) & 0.28 & 0.02 \\
\hline Left IFG (pars triangularis) & 0.35 & 0.004 & 0.04 \\
\hline Right IFG (pars triangularis) & 0.43 & 0.01 & 0.02 \\
\hline Left STG (planum temporale) & 0.39 & 0.12 & 0.03 \\
\hline Right STG (planum temporale) & 0.25 & 0.12 \\
\hline IFG: inferior frontal gyrus; STG: superior temporal gyrus; FDR; false discovery rate & & \\
\hline
\end{tabular}




\section{Figures}

a

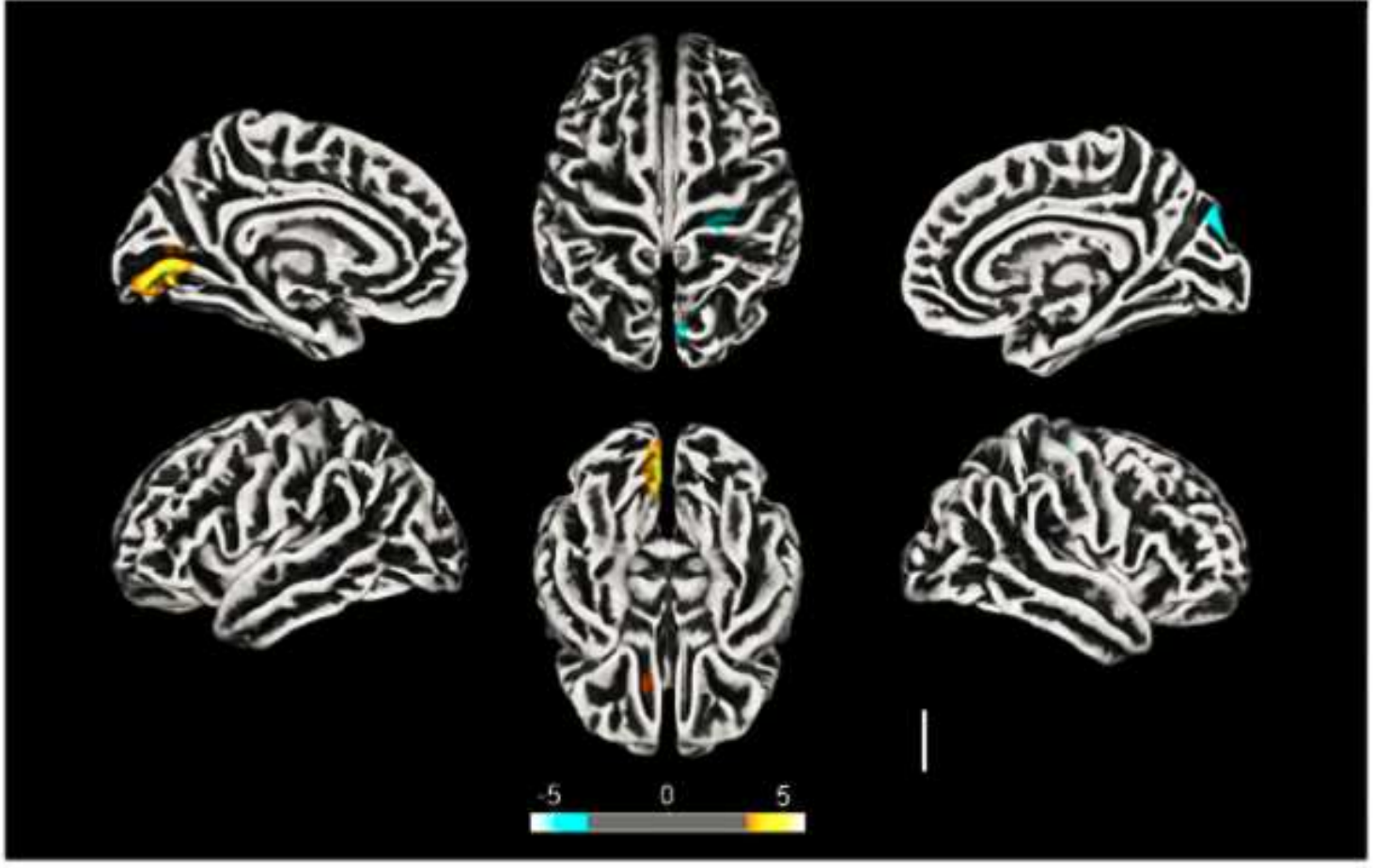

b
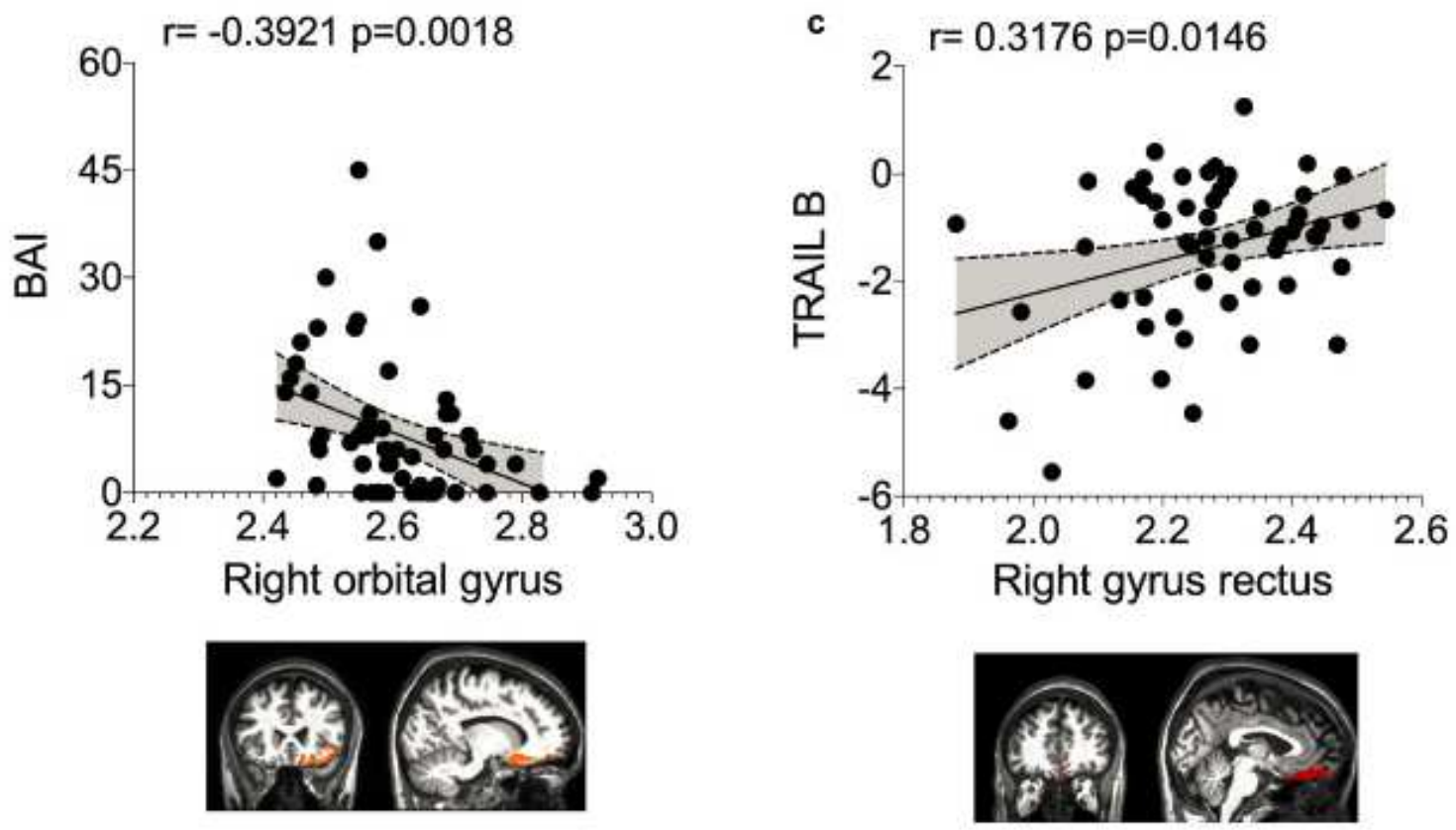

Figure 1

Altered cerebral cortical thickness is associated with neuropsychiatric symptoms in COVID-19 patients. a) Surface-based morphometry using high-resolution 3T MRI. Yellow represents areas of decreased cortical thickness: left lingual gyrus, calcarine sulcus (and cuneus), and olfactory sulcus (and rectus gyrus). Blue 
represents areas of increased cortical thickness: central sulcus (precentral and postcentral gyrus) and superior occipital gyrus. Representative image of the analysis of 81 subjects tested positive for SARSCoV-2 (who had mild respiratory symptoms and did not require hospitalization or oxygen support) compared to 145 healthy volunteers (without diagnosis of COVID-19). The analysis was performed within a median interval of 54 days. b) Correlation between anxiety scores (BAI) and right orbital gyrus thickness. The data depicts Pearson's correlation coefficient. c) Correlation between TRAIL B performance scores and right gyrus rectus thickness. Data depict Pearson's correlation coefficient and region of interest in representative images. 
a

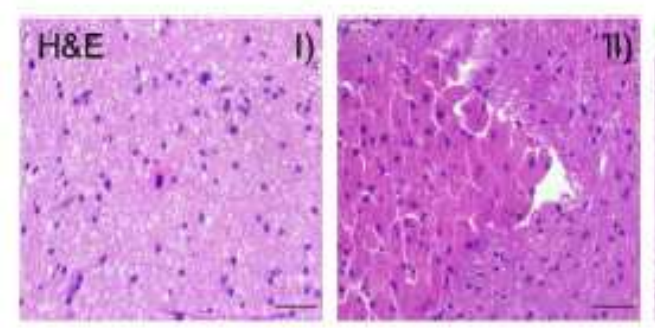

c
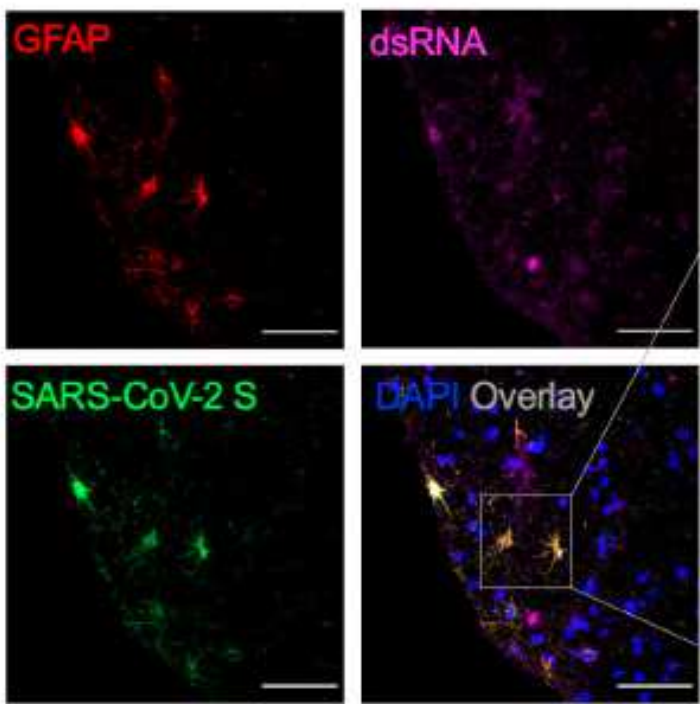

III).
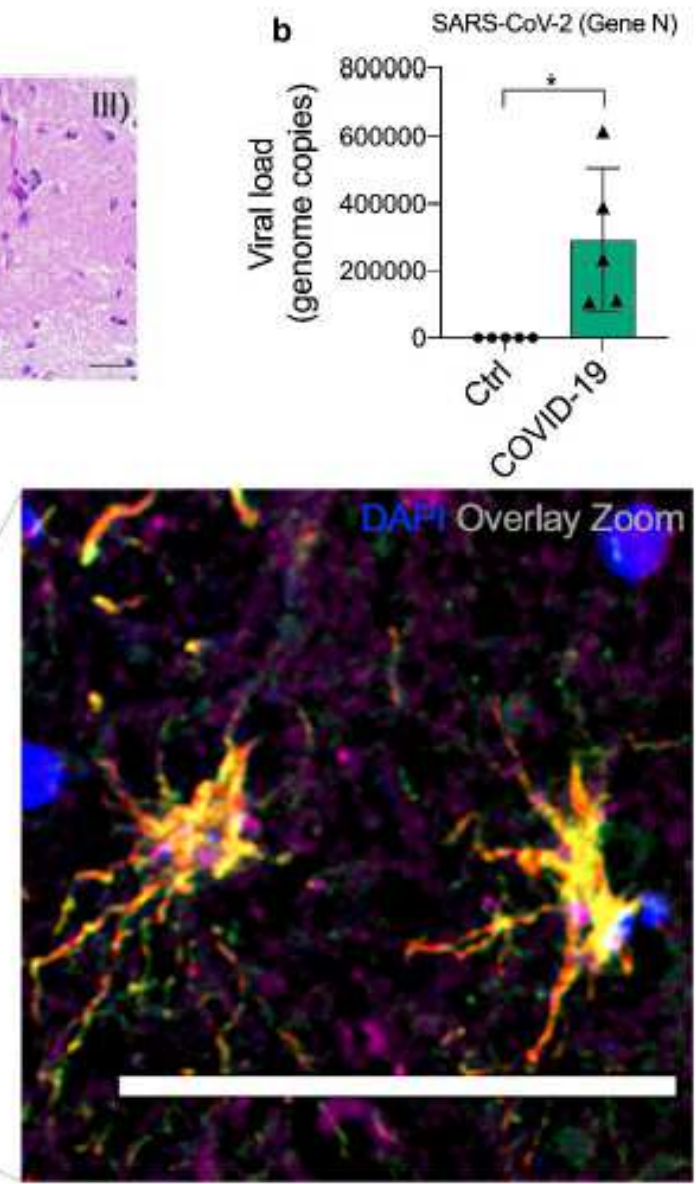

e SARS-CoV-2-S ${ }^{+}$cells
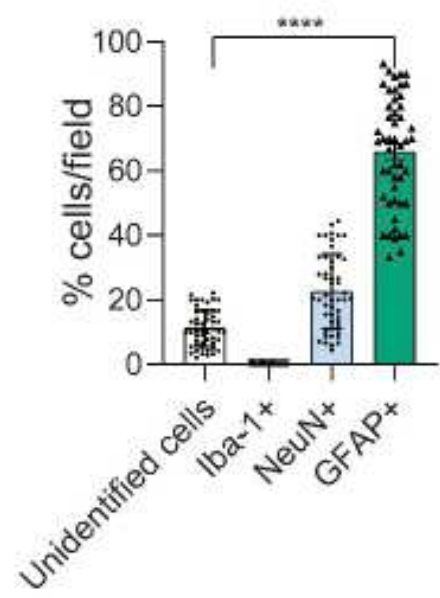

f

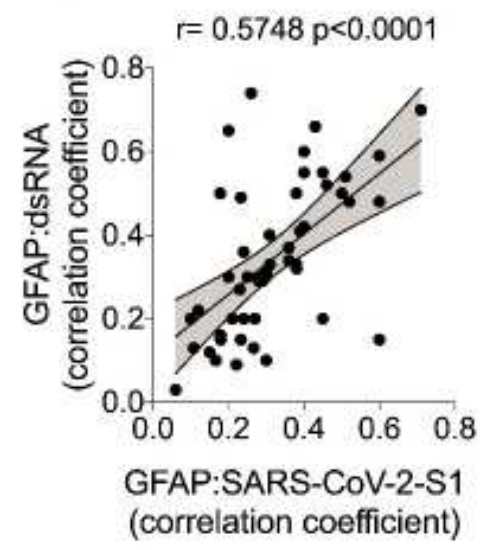

\section{Figure 2}

SARS-CoV-2 infects the central nervous system, replicates in astrocytes and causes brain damage. a) Histopathological H\&E images of postmortem brain tissue from individuals who died of COVID-19. Five out of 26 individuals showed signs of brain damage as represented in the images by I) areas of necrosis, cytopathic damage (I, i.e., enlarged, hyperchromatic, atypical-appearing nuclei), vessels with margination of leukocytes and thrombus (II), and immune cells infiltrate (III). Images were acquired with 400x 
magnification. Scale bar indicates $50 \mu \mathrm{m}$. b) Viral load in brain tissues from the five COVID-19 patients who manifested histopathological alteration in the brain as compared to samples from SARS-CoV-2negative controls ( $n=5$ per group). c) Representative confocal images of the brain tissue of one COVID-19 patient who manifested histopathological alterations. Immunofluorescence targeting glial fibrillary acidic protein (GFAP, red), double-stranded RNA (dsRNA, magenta), SARS-CoV-2-S (green), and nuclei (DAPI, blue). Images were acquired with 630x magnification. Scale bar indicates $50 \mu \mathrm{m}$. d) Percentage of SARSCoV-2-S positive cells in this tissue. e) Percentage of GFAP-positive vs. Unidentified cells, Iba1 and NeuN among infected cells. Ten fields/cases were analyzed. f) Pearson's correlation coefficient demonstrating colocalization of SARS-CoV-2-S and dsRNA within GFAP-positive cells. Data shown as mean \pm SEM. Pvalues were determined by two-tailed unpaired tests with Welch's correction (b) or ANOVA one-way followed by Tukey's post hoc test (e). ${ }^{\star} P<0.05$ compared to the Ctrl-group; ${ }^{* \star \star \star} P<0.0001$ compared to Unidentified cells, Iba1 and NeuN groups. 
a

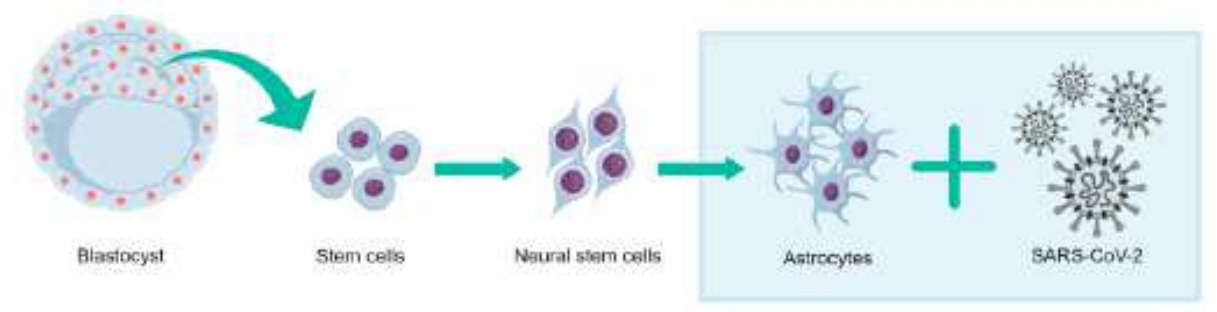

b
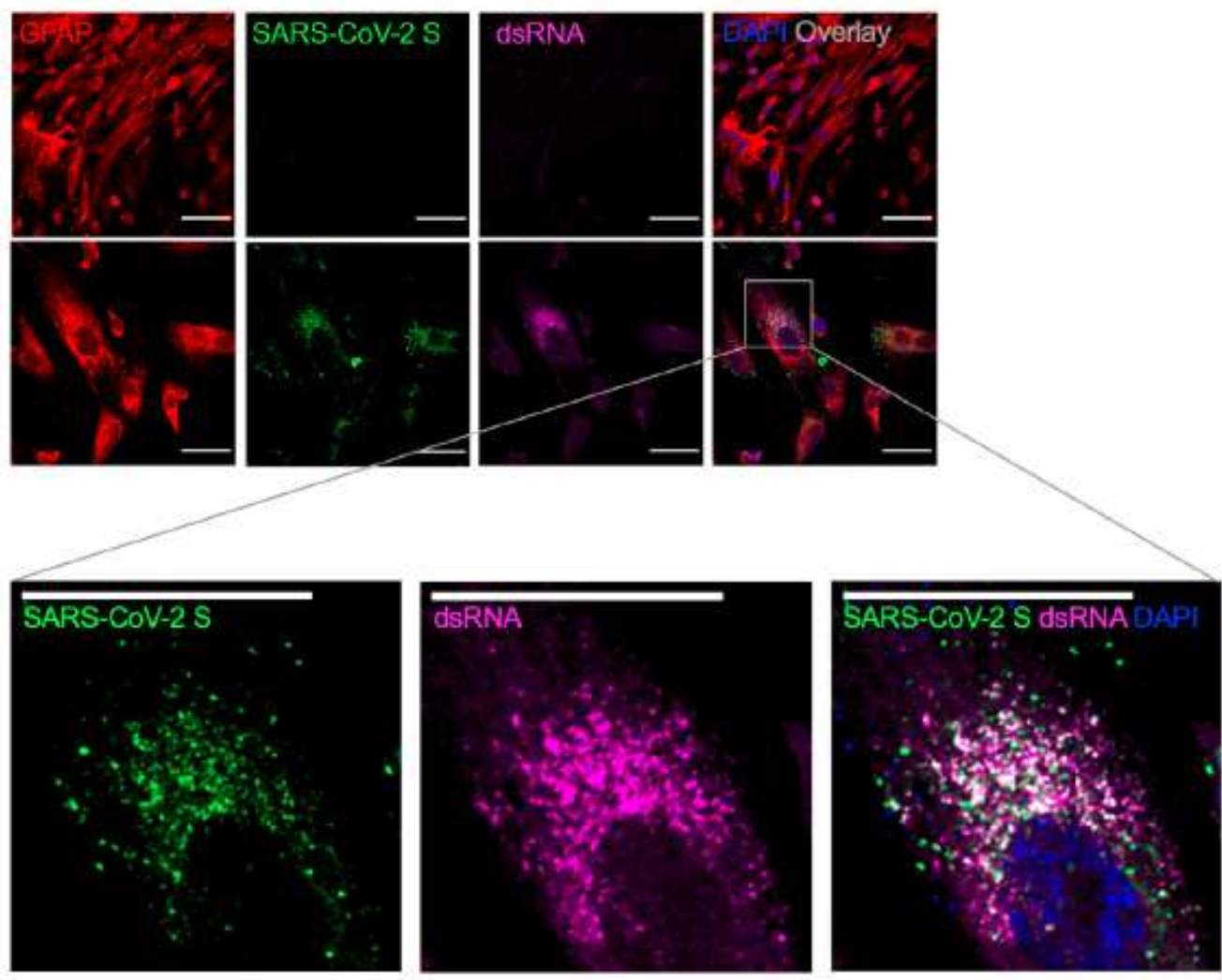

c

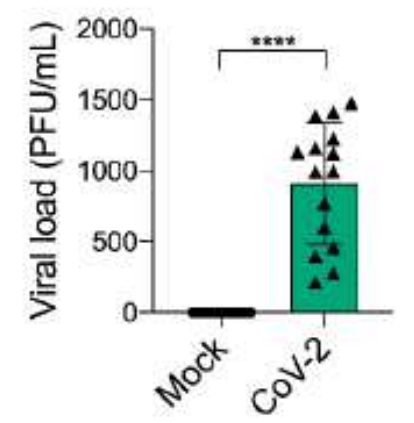

d Astrocytes

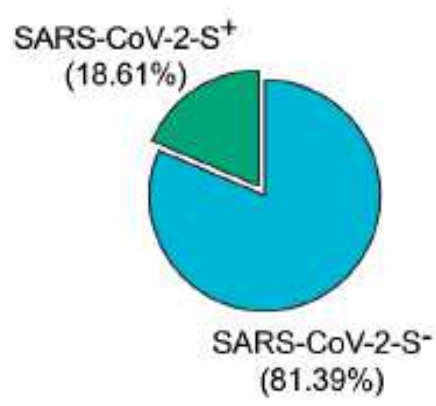

- SARS-Cov-2-S cells

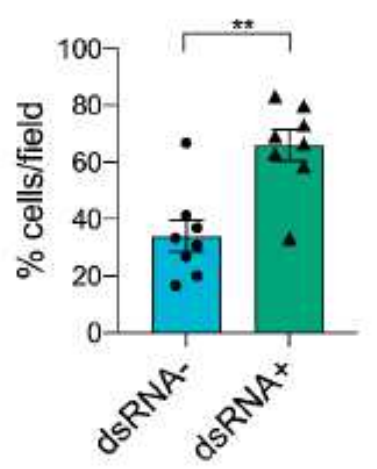

\section{Figure 3}

SARS-CoV-2 infects and replicates in astrocytes in vitro. a) Human neural stem cell-derived astrocytes were infected in vitro with SARS-CoV-2 (MOI 1) for $1 \mathrm{~h}$, washed, and harvested $24 \mathrm{~h}$ after infection. $\mathrm{b}$ ) Immunostaining for GFAP (red), double-stranded RNA (dsRNA, magenta), SARS-CoV-2-S (green), and nuclei (DAPI, blue). Images were acquired with 630x magnification. Scale bar indicates $50 \mu \mathrm{m}$. c) SARSCoV-2 viral load detection in astrocyte cell pellet ( $n=15$ replicates) using RT-PCR. $d$ ) Percentage of 
infected astrocytes. The data depicts SARS-CoV-2-S and DAPI stained cells (100 fields were analyzed). e) Frequency of cells containing replicating viruses. The data represents the percentage of dsRNA-stained cells into SARS-CoV-2-S positive cells (10 fields were analyzed). Data are representative of at least two independent experiments and shown as mean \pm SEM. P-values were determined by two-tailed unpaired tests with Welch's correction ( $b$ and $d$ ). ${ }^{\star *} \mathrm{P}<0.01 ;{ }^{* \star \star *} \mathrm{P}<0.0001$ compared to the mock group.

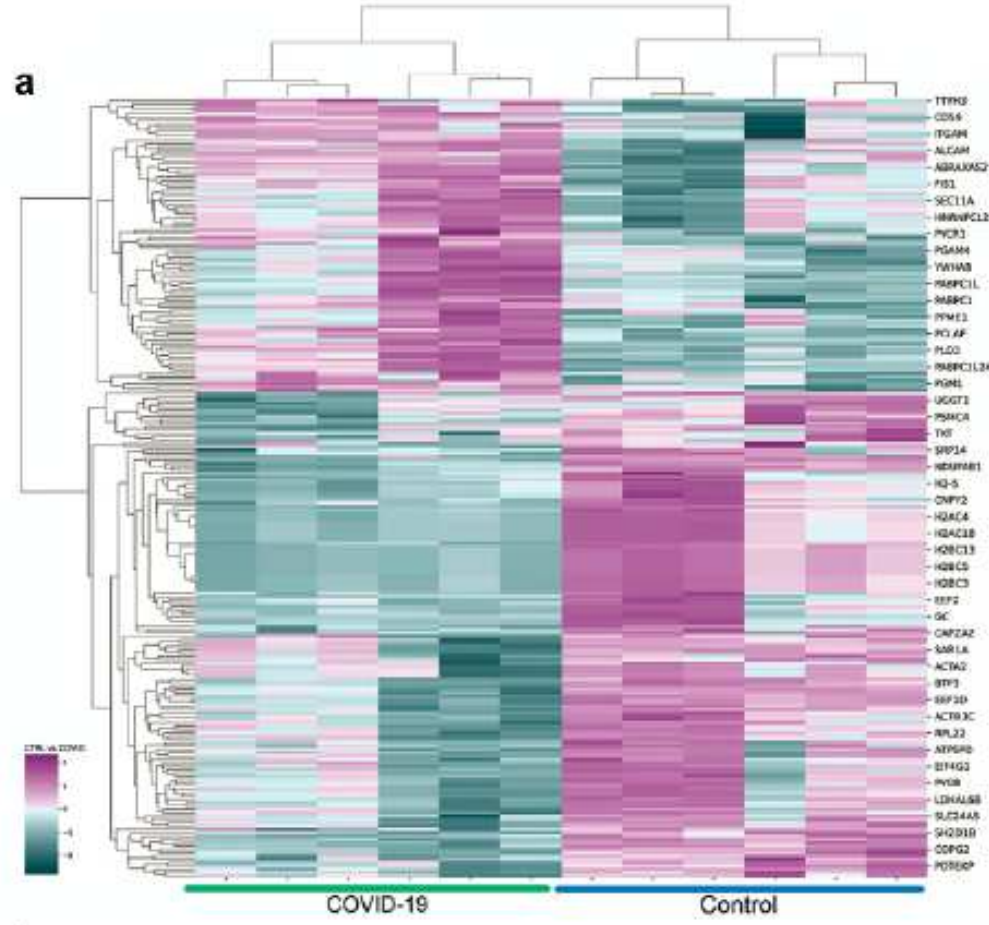

b

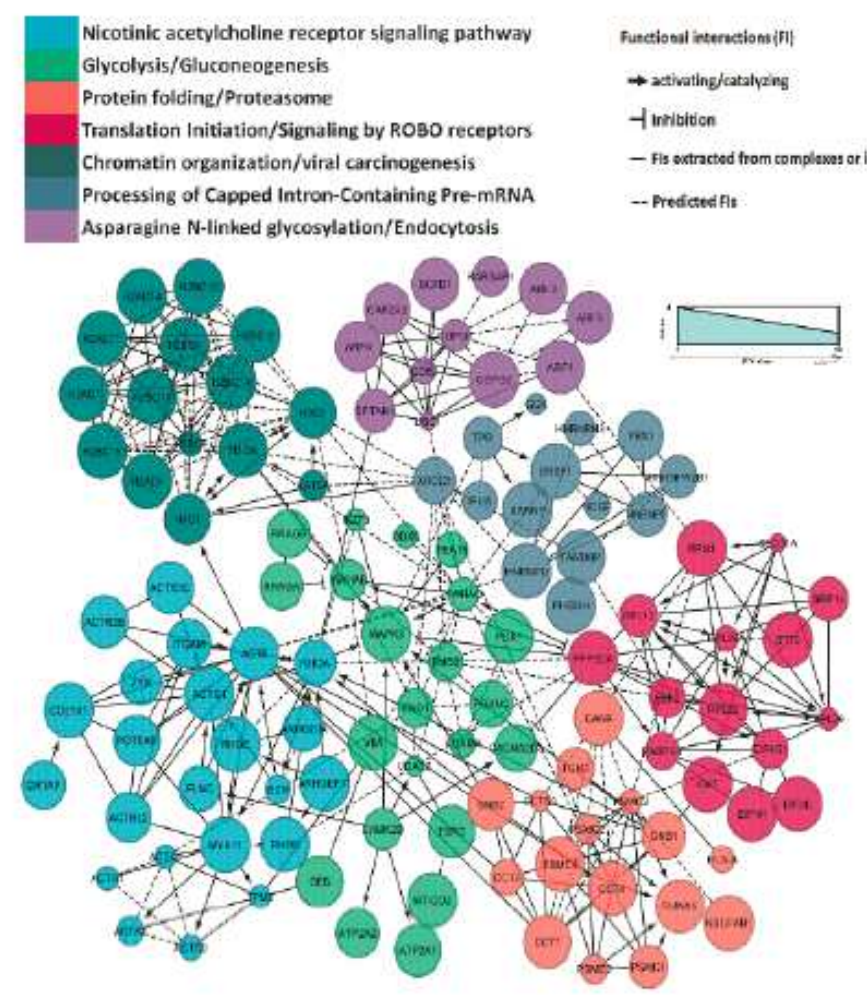

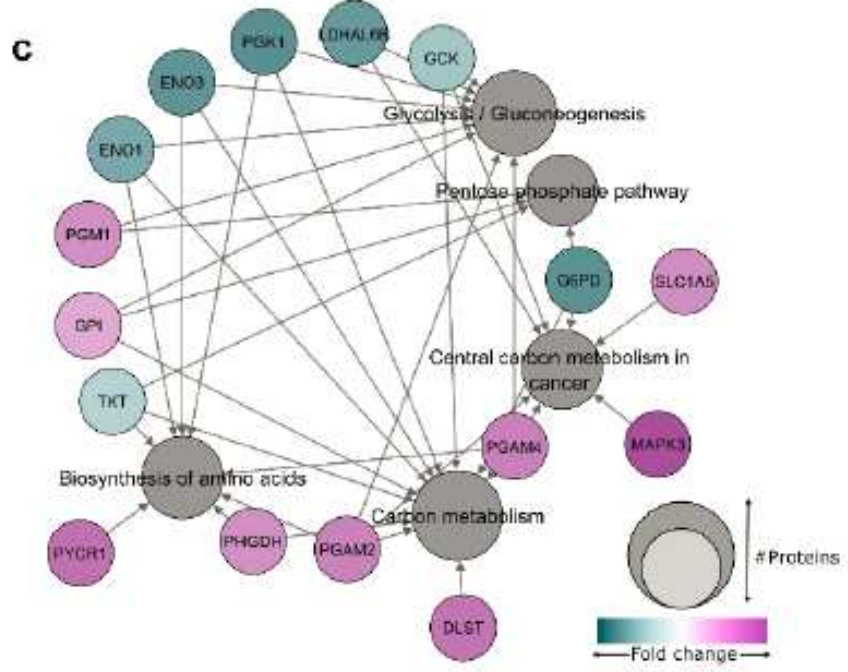

d

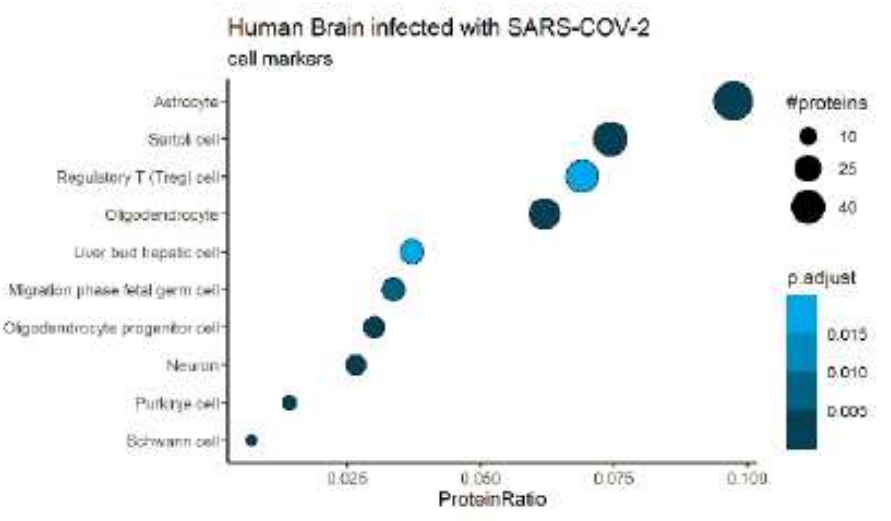

e

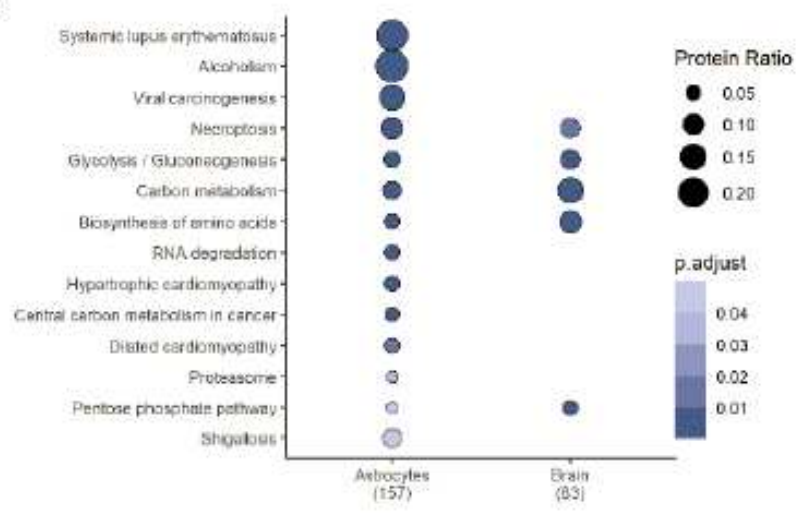

Figure 4 
Proteomic changes in SARS-CoV-2-infected human astrocytes and postmortem brain tissue from COVID19 patients a) Hierarchical clustering of differentially expressed proteins of human neural stem cellderived astrocytes were infected in vitro with SARS-CoV-2 (MOI 0.1) for $1 \mathrm{~h}$, washed thoroughly and harvested after $24 \mathrm{~h}$. Mock was used as a control. b) Reactome functional interaction network of differentially regulated genes of human neural stem cell-derived astrocytes were infected in vitro with SARS-CoV-2. Seven different colors show 7 protein clusters of enriched pathways specified by color and the arrows represents the protein-protein interaction and the downstream activation or inhibition related with the gene modulation, showing how some pathways can be affected by SARS-CoV- 2 infection $(p<0.05$ calculated based on binomial test). $c$ ) Network of proteins found differentially regulated in SARSCOV-2-infected Astrocytes and their respective pathways, enriched according to KEGG database. The pathways are represented by gray circles and their size is proportional to the number of proteins differentially regulated; proteins are represented as the smallest circles, colored according to their fold change. d) Cell type-enrichment analysis using the dataset generated by postmortem brain tissue from patients who died of Covid-19. Dot size represents the number of proteins related to the respective cell type and the $p$ value adjusted by the false discovery rate (FDR). e) KEGG-enrichment analysis of differentially expressed proteins in SARS-CoV-2 infected astrocytes vs. mock as compared to postmortem brain tissue from COVID-19 patients vs. controls. Dot size represents the number of proteins related to the respective cell type and the $p$ value adjusted by the false discovery rate (FDR). 
a

Pyruvate

Lactate

Glutamine

Glutamate

GABA

$\alpha$-ketoglutarate
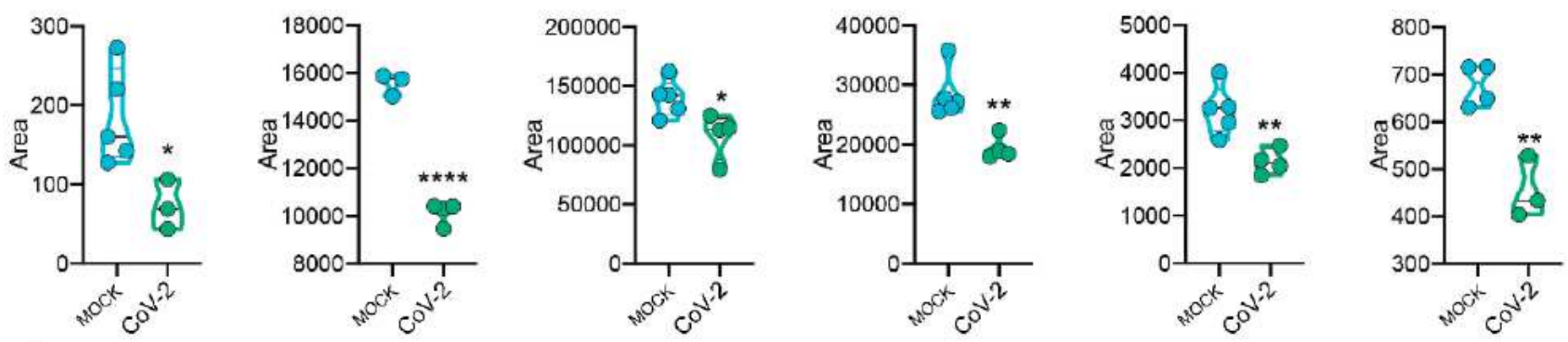

b

C
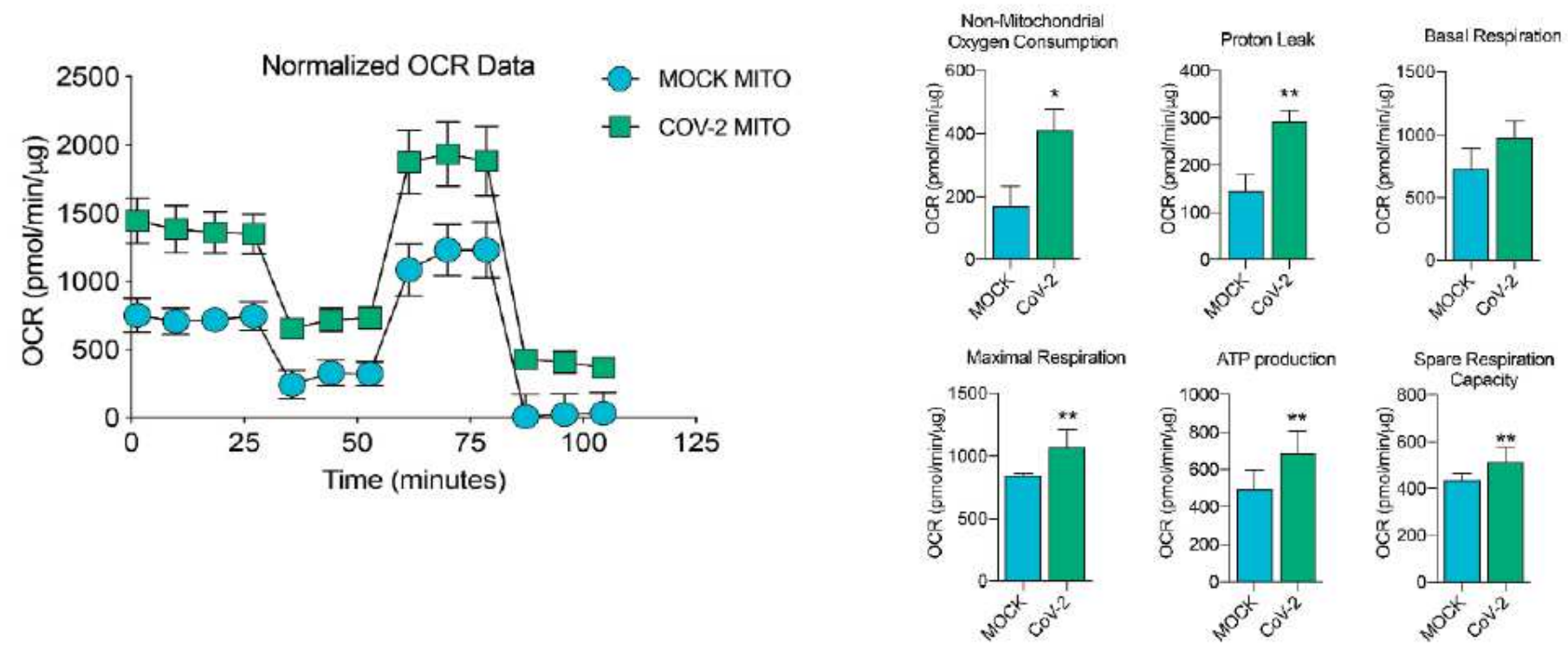

Figure 5

SARS-CoV-2 infection alters astrocyte metabolism. Human neural stem cell-derived astrocytes were infected in vitro with SARS-CoV-2 (MOI 0.1) for $1 \mathrm{~h}$, washed thoroughly and harvested after $24 \mathrm{~h}$. Mock was used as a control. a) High-resolution mass spectrometry quantification of pyruvate, lactate, glutamine, glutamate, GABA, and a-ketoglutarate in SARS-CoV-2 infected astrocytes vs. mock. The integration area of each peak was used to calculate the violin plot graph. b) Oxygen consumption rate (OCR) of SARS-CoV-2 infected astrocytes vs. mock. SeaHorse Flux Analysis using the MitoStress test where basal respiration was measured followed by determination of oligomycin-, FCCP-, and rotenone/antimycin-induced respiration. Data are representative of at least two independent experiments performed in triplicate (metabolomics analysis) or quintuplicate (SeaHorse Flux Analysis) and shown as mean \pm SEM. $P$ values were determined by two-tailed unpaired with Welch's correction. *P $<0.05$; ${ }^{*} \mathrm{P}<$ $0.01 ; * \star * \star \mathrm{P}<0.0001$ compared to mock. 
a

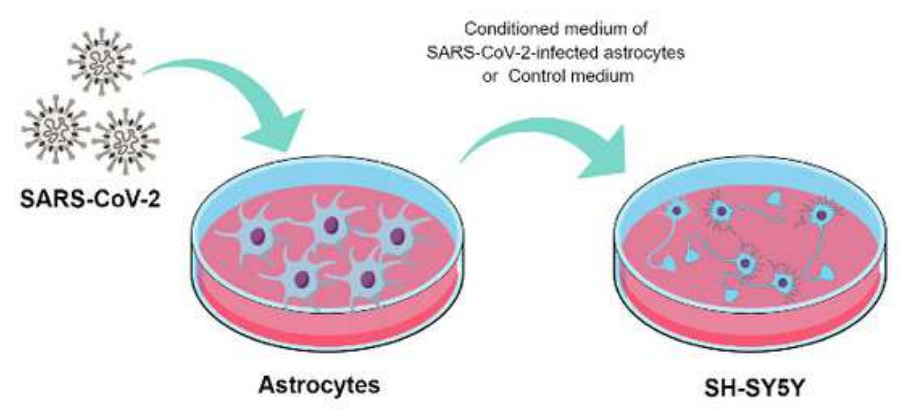

b

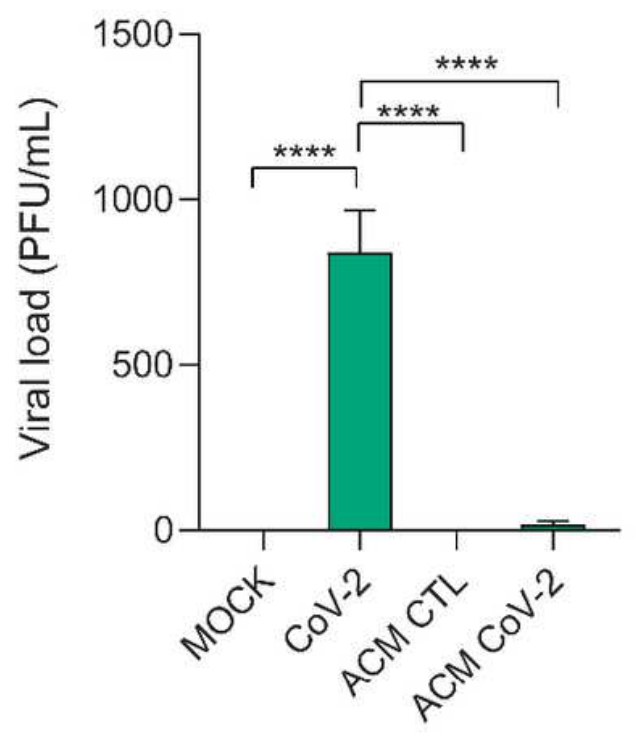

C

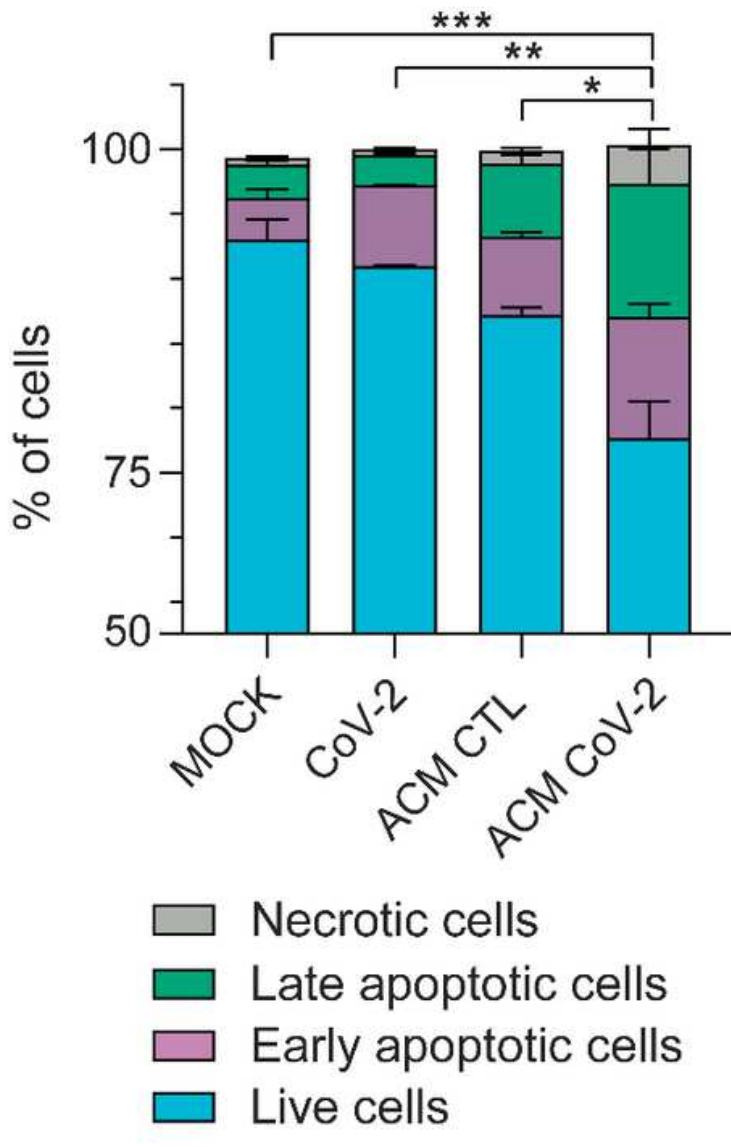

\section{Figure 6}

Conditioned medium of SARS-CoV-2-infected astrocytes reduces neuronal viability. a) Human neuronal cell line differentiated SH-SY5Y was cultured for $24 \mathrm{~h}$ in the presence of the conditioned medium of SARS-CoV-2-infected astrocytes from mock (ACM CTL) or SARS-CoV-2 (ACM CoV-2) infected cells. a) SARS-CoV-2 viral load detection in differentiated SH-SY5Y neurons cell pellet using RT-PCR. b) Cellular viability of SH-SY5Y incubated for 24 hours with conditioned medium of SARS-CoV-2-infected astrocytes (ACM) from mock (ACM CTL) or SARS-CoV-2 (ACM CoV-2) infected cells by apotracker/fixable viability stain (FVS) staining. Live (dark blue) represents double-negative population, early apoptosis (purple) and late apoptosis (green) show apotracker+FVS- and double-positive cells, respectively, and necrosis (gray) is apotracker-FVS+. Data are representative of at least two independent experiments performed in triplicate and shown as mean \pm SEM. P values were determined by one-way ANOVA followed by Tukey's post. 